\title{
Spatial localization in heterogeneous systems
}

\author{
Hsien-Ching Kao* \\ Wolfram Research Inc., Champaign, Illinois 61820, USA \\ Cédric Beaume ${ }^{\dagger}$ and Edgar Knobloch ${ }^{\ddagger}$ \\ Department of Physics, University of California, Berkeley, California 94720, USA
}

(Received 18 October 2013; published 6 January 2014)

\begin{abstract}
We study spatial localization in the generalized Swift-Hohenberg equation with either quadratic-cubic or cubic-quintic nonlinearity subject to spatially heterogeneous forcing. Different types of forcing (sinusoidal or Gaussian) with different spatial scales are considered and the corresponding localized snaking structures are computed. The results indicate that spatial heterogeneity exerts a significant influence on the location of spatially localized structures in both parameter space and physical space, and on their stability properties. The results are expected to assist in the interpretation of experiments on localized structures where departures from spatial homogeneity are generally unavoidable.
\end{abstract}

DOI: 10.1103/PhysRevE.89.012903

PACS number(s): 82.40.Ck, 47.54.-r, 47.20.Ky, 47.55.P-

\section{INTRODUCTION}

A large variety of physical systems exhibits stationary spatially localized structures in appropriate parameter regimes. These include different convective systems driven by an imposed temperature difference [1-7], a ferrofluid subject to an imposed magnetic field [8] and an optical light valve experiment driven by a nominally uniform light intensity [9]. Other systems exhibiting localized structures include shear flows [10,11], gas discharges [12], and a variety of optical configurations $[13,14]$. In modeling these systems one typically assumes that the forcing of the system is spatially homogeneous, be it the imposed temperature difference across a convection system or the magnetic field imposed across a ferrofluid. In experiments, however, spatial homogeneity is difficult if not impossible to achieve. Spatial heterogeneities arise from a variety of sources, including edge or sidewall effects, imperfect temperature control, magnetic field perturbations, and a variety of topographic effects such as surface imperfections that are usually assumed to be absent. Lateral parameter gradients generally lead to drift [15] and trapping of structures by spatial heterogeneities in the gradients. In the present work we study the steady structures created by these processes with a view to gaining a solid understanding of the effects of spatial heterogeneities on the presence and stability of stationary localized structures. We show, in particular, that spatial heterogeneities may, under appropriate conditions, substantially modify the standard homoclinic snaking scenario [16] that has been so successful in interpreting the results of numerical simulations of nominally one-dimensional systems $[4,17]$.

The Swift-Hohenberg equation has played a fundamental role in our understanding of homogeneous systems, serving as a "normal form" for systems undergoing a steady-state instability with a finite wave number at onset. The reason for this is that in one spatial dimension the basic snakes-and-ladders structure [18] of the so-called snaking or pinning region in

\footnotetext{
*hkao@wolfram.com

†ced.beaume@gmail.com

${ }^{\ddagger}$ knobloch@berkeley.edu
}

parameter space that organizes the localized structures in two or four intertwined solution branches is generated by transversal intersections of certain manifolds [19,20]. Such intersections are structurally stable with respect to sufficiently small changes in the equation such as changes in the parameter values or in the form of the nonlinearity. The equation takes the form

$$
u_{t}=a u-\left(1+\partial_{x x}\right)^{2} u+N(u)
$$

where $u(x, t)$ is a scalar order parameter, $x$ and $t$ denote space and time variables, and $N(u)$ is a smooth nonlinear function. The parameter $a$ represents forcing, with the trivial state $u=0$ stable for $a<0$ and unstable for $a>0$. Note that $x$ has been scaled so that the primary instability corresponds to wave number $k=1$. With periodic boundary conditions on the spatial domain $[-\Gamma / 2, \Gamma / 2]$, Eq. (1) exhibits variational dynamics with the Lyapunov functional (or free energy)

$$
\mathcal{F} \equiv \int_{-\Gamma / 2}^{\Gamma / 2}\left\{-\frac{1}{2} a u^{2}+\frac{1}{2}\left[\left(1+\partial_{x x}\right) u\right]^{2}-\int_{0}^{u} N(v) d v\right\} d x .
$$

This property implies that any initial condition integrated forward in time tends, as time increases, to either a stationary state or a front propagating at constant speed. We define the Maxwell point as the value of the parameter $a$ for which $\mathcal{F}=0$ along the branch of spatially periodic solutions emerging from the primary instability at $a=0$. At this value of $a$ the periodic state with wave number $k=1$ has the same free energy as the trivial state $u=0$. Two choices of $N(u)$ have proved particularly useful:

$$
\begin{gathered}
N_{23}(u) \equiv 1.8 u^{2}-u^{3}, \\
N_{35}(u) \equiv 2 u^{3}-u^{5}
\end{gathered}
$$

Hereafter, we refer to the Swift-Hohenberg equation with the nonlinearity $N_{23}$ (resp. $N_{35}$ ) as SH23 (resp. SH35). In both cases, Eq. (1) is translation invariant in $x$ and spatially reversible with respect to an arbitrary origin, here chosen to 
be $x=0$, i.e., under the operation

$$
R_{1}: x \rightarrow-x, \quad u \rightarrow u .
$$

The choice $N_{35}$ introduces the additional symmetry

$$
R_{2}: x \rightarrow x, \quad u \rightarrow-u .
$$

This symmetry is appropriate for modeling physical systems with midplane reflection symmetry such as Boussinesq convection or plane Couette flow.

In the following we generalize the Swift-Hohenberg equation and incorporate spatially heterogeneous conditions by allowing the parameter $a$ to depend on $x$. This assumption destroys the translation invariance and selects preferred locations in space for the localized structures that remain. Of course translation invariance may be broken in any number of different ways (the translation invariant problem is formally of infinite codimension), but we choose here two representative forcing profiles, $a=a(x) \equiv r[1+\alpha f(x)]$, where $f(x)$ is either periodic with period $2 \pi / \delta$ or Gaussian with width $\sigma$ :

$$
f_{p}(x) \equiv \cos (\delta x), \quad f_{b}(x) \equiv \exp \left(-x^{2} / 2 \sigma^{2}\right)
$$

The periodic heterogeneity $f_{p}$ corresponds to a sinusoidal forcing with mean $r$, relative oscillation amplitude $\alpha$, and wave number $\delta$. The case $\delta=1$ corresponds to a $1: 1$ resonance between the wave number of the forcing and that intrinsic to the Swift-Hohenberg equation. The bump heterogeneity $f_{b}$ models homogeneous forcing locally perturbed by a bell-like departure. In this case, the background forcing has amplitude $r$ and the bump is modeled using a Gaussian function of amplitude $\alpha$ and width proportional to $\sigma$.

In the following sections, we report results on the location and stability of localized structures in the presence of the heterogeneities $f_{p}$ and $f_{b}$ with different spatial scales and amplitude. The numerical continuation package AUTO [21] is used to follow solutions in a domain of spatial extent $\Gamma=40 \pi$, a length sufficiently large (20 times the critical wavelength) to allow spatial localization. Owing to the choice of forcing, the system is spatially reversible with respect to $x=0$ and we carry out our computations on the half-domain $[0, \Gamma / 2]$. The solutions for $\mathrm{SH} 23$ are computed using Neumann boundary conditions

$$
u_{x}=u_{x x x}=0
$$

at $x=0, \Gamma / 2$ and then reflected in $x=0$ to obtain solutions on the full domain. For SH35, this procedure yields only solutions with even parity. To find solutions with odd parity we impose Dirichlet boundary conditions

$$
u=u_{x x}=0
$$

at $x=0, \Gamma / 2$ and use the symmetry $R_{1} \circ R_{2}$ to extend the solution to the full domain. In all cases we show bifurcation diagrams in terms of the $L_{2}$ norm of the solution, defined as

$$
\|u\|_{L_{2}} \equiv\left(\frac{1}{\Gamma} \int_{-\Gamma / 2}^{\Gamma / 2} u^{2} d x\right)^{1 / 2}
$$

In the following section, we recall the basic results for the formation of localized structures in the Swift-Hohenberg equation with homogeneous forcing. In Sec. III we present results obtained when a periodic heterogeneity with $O(1)$ length scale is turned on, i.e., when the length scale of the heterogeneity is comparable to the natural length scale of the problem. Section IV is devoted to the stability properties of the solutions subject to heterogeneous forcing. Section V presents results for heterogeneities that vary on the scale $O(\Gamma)$, i.e., on the scale of the domain size, with $\Gamma \gg 1$. In each case we examine the effects of periodic forcing with the requisite scale and compare it with the effects of an isolated Gaussian bump with a comparable length scale. In Sec. VI we determine the displacement of the saddle nodes of the competing periodic branches through an analytical calculation, followed in Sec. VII by an investigation of temporal dynamics of spatially localized patterns in the presence of heterogeneous forcing. The paper concludes with a summary of the results together with a discussion of their implications for experiments.

\section{HOMOGENEOUS FORCING}

The homogeneous one-dimensional Swift-Hohenberg equation has been the focus of a number of recent studies $[16,18,20,22]$ and the formation of localized structures in one spatial dimension within this equation is now well understood. Here we provide a brief overview of known results for $\mathrm{SH} 23$ and $\mathrm{SH} 35$ that will be used for comparison with the new results in the following sections. The results in the presence of homogeneous forcing for SH23 and SH35 are reported in Figs. 1 and 2, respectively. The trivial solution first becomes unstable at $r=0$ due to a subcritical bifurcation. This bifurcation creates a one-parameter family of spatially periodic states of wavelength $2 \pi$. At each $r$ we select two representatives from this family. In $\mathrm{SH} 23$ one of the solutions has a peak at $x=0$ (hereafter $\phi=0$, black curve), while the other has a trough at $x=0$ [hereafter $\phi=\pi$, red (or gray) curve]. Both are of even parity. In SH35 these solutions are related by the additional $R_{2}$ symmetry and are both represented in black. However, one also has odd solutions with $\left.u\right|_{x=0}=0$ and either a positive $(\phi=\pi / 2)$ or a negative $(\phi=3 \pi / 2)$ slope at $x=0$. These solutions are referred to as odd parity solutions and are represented in red (or gray). This color convention will be used throughout the remainder of this paper. In both cases, the spatially periodic branches undergo saddle node bifurcations before turning towards larger values of $r$ and hence larger amplitudes. Owing to the finite size of the domain, a modulational instability occurs along these branches at small but nonzero amplitude [23] generating two distinct branches of localized states: the $\phi=0$ and $\phi=\pi$ branches in SH23, and two even $(\phi=0, \pi)$ and two odd $(\phi=\pi / 2,3 \pi / 2)$ branches in $\mathrm{SH} 35$, each arising from modulational instability occurring along the periodic solutions of the appropriate phase. All these localized branches exhibit back-and-forth oscillations within a well-defined parameter interval in a behavior known as homoclinic snaking [19]. These oscillations reflect the nucleation of additional oscillations in the solution profile at the locations of the fronts bounding the structure, as described in greater detail in Ref. [18], resulting in the growth of the structure and hence increased $L_{2}$ norm. In SH23 the solution adds one oscillation on either side after every second saddle node until the solution fills the domain and the solution branch exits the snaking region. In $\mathrm{SH} 35$ the process is similar but the 
(a)

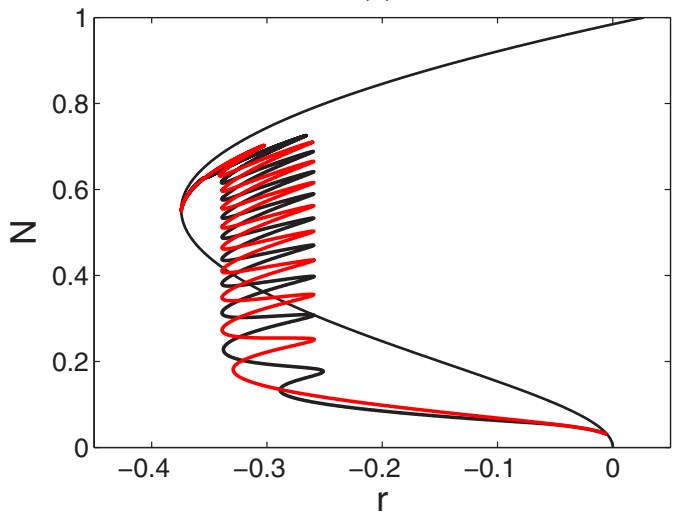

(b)

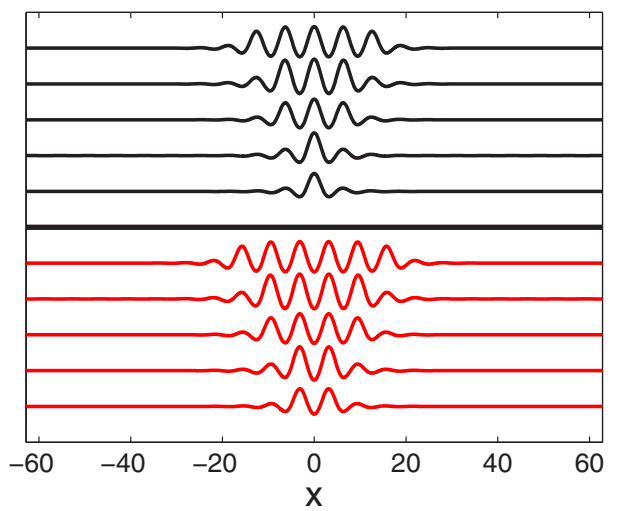

FIG. 1. (Color online) (a) Bifurcation diagram showing $N \equiv$ $\|u\|_{L_{2}}$ as a function of $r$ for homogeneous forcing $(\alpha=0)$ in SH23. The black [red (or gray)] snaking branch corresponds to solutions with $\phi=0(\phi=\pi)$. (b) Solution profiles at the bottom five saddle nodes along each snaking branch. The asymmetric rung states [22] are omitted.

solution adds half of an oscillation (either a peak or a trough) between every second saddle node implying that the frequency of the back-and-forth oscillations of the branch is doubled. Once the branch of localized states exits the snaking region it reconnects with the original periodic branch near its saddle node. During the snaking, all the branches of localized states experience alternating stability and instability as the amplitude eigenvalue oscillates through zero at successive saddle nodes. As a result the segments of each branch connecting the saddle nodes from left to right (proceeding upwards) are stable and the rest is unstable. The resulting diagrams constitute reference results for the subsequent sections on spatially dependent forcing.

We mention that the branches of even states in $\mathrm{SH} 23$ are connected by a series of rungs consisting of asymmetric states, forming a snakes-and-ladders structure [22]. Similar rungs connect even and odd states in SH35 [16,18]. All are unstable, but will be found to play a significant role once $\alpha \neq 0$.

\section{HETEROGENEOUS FORCING ON A $O(1)$ SPATIAL SCALE}

In this section we examine the effects of spatially periodic forcing on a $O(1)$ spatial scale. The values of the parameters (a)

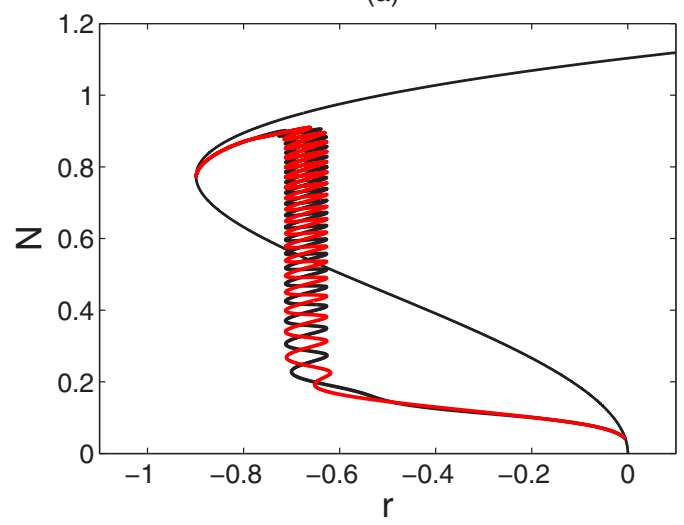

(b)

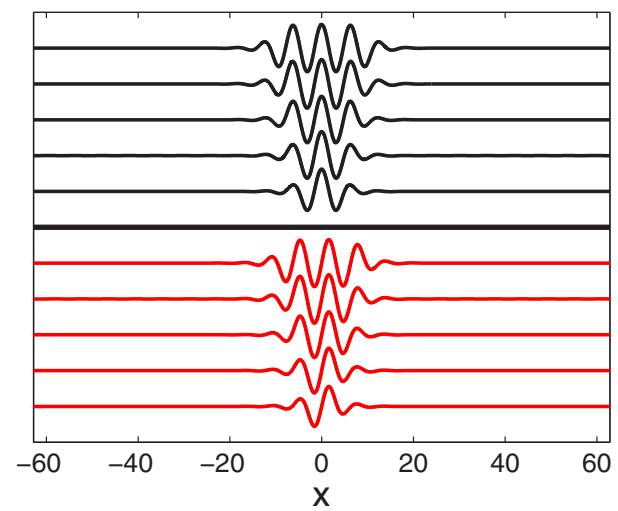

FIG. 2. (Color online) (a) Bifurcation diagram showing $N \equiv$ $\|u\|_{L_{2}}$ as a function of $r$ for homogeneous forcing $(\alpha=0)$ in SH35. The black [red (or gray)] snaking branch corresponds to even (odd) solutions. (b) Solution profiles at the bottom five saddle nodes along the left end of the snaking branches. The asymmetric rung states [18] are omitted.

are $\delta=1$ for the periodic heterogeneity $f_{p}$ and $\sigma=\pi / 2$ for the bump heterogeneity $f_{b}$.

\section{A. Periodic heterogeneity $f_{p}$}

Here we present the results when the heterogeneity $f_{p}$ is turned on. Bifurcation diagrams obtained with the quadraticcubic nonlinearity are shown in Fig. 3 for $\alpha= \pm 0.1$. We consider two branches of symmetric periodic states, those in-phase $(\Delta \phi=0)$ and those out-of-phase $(\Delta \phi=\pi)$ with the forcing. Thus the maxima (minima) of the $\Delta \phi=0(\Delta \phi=\pi)$ states coincide with the forcing maxima. As seen in the figure the $\Delta \phi=0, \pi$ branches differ. When $\alpha>0$, the saddle node of the $\Delta \phi=0$ periodic branch [black curve in Fig. 3(a)] moves further to the left beyond its original location, extending the region of bistability and allowing the $\Delta \phi=0$ localized states to extend further into the subcritical regime. Figure 3(a) shows the complete snaking branch of the resulting $\Delta \phi=0$ localized states [solid red (or gray) curve]; the branch resembles qualitatively the snaking branch of the $\phi=\pi$ states familiar from SH23 with homogeneous forcing (Fig. 1). In contrast, the saddle node of the $\Delta \phi=\pi$ periodic branch [black curve in Fig. 3(b)] shifts towards the right as $\alpha$ increases, reducing the region of bistability and hence the width of the snaking 
(a)

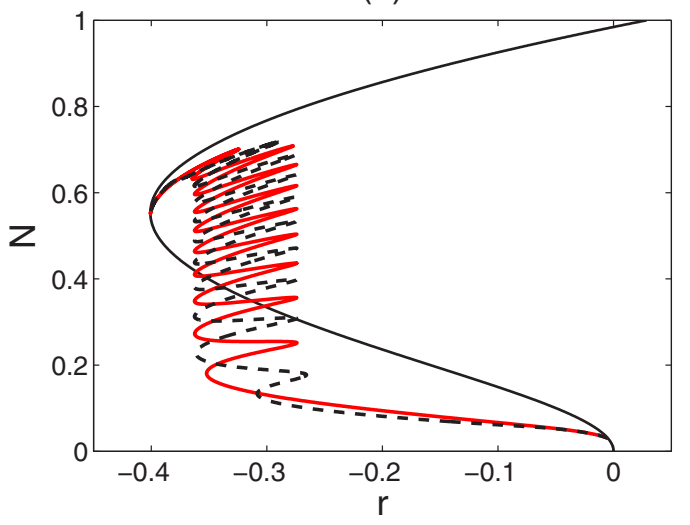

(b)

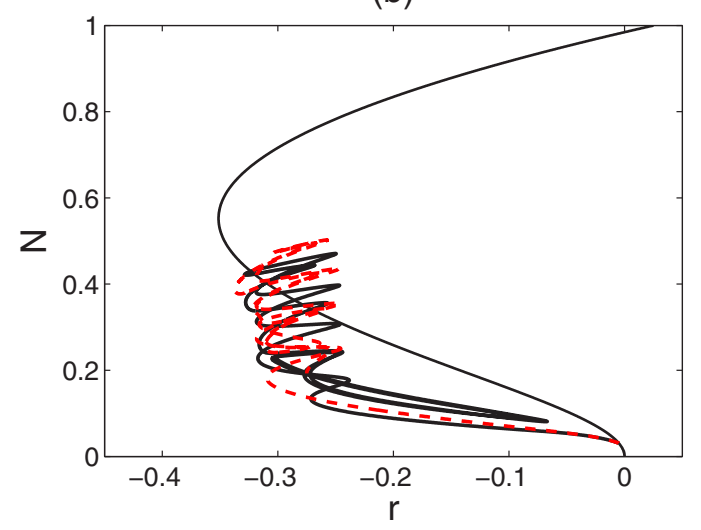

FIG. 3. (Color online) Bifurcation diagrams for $\mathrm{SH} 23$ with the forcing $f_{p}$ and $\delta=1$, showing $N \equiv\|u\|_{L_{2}}$ as a function of $r$ for (a) $\Delta \phi=0$ steady states and (b) $\Delta \phi=\pi$ steady states. The forcing amplitude $\alpha=0.1$ (solid lines) and $\alpha=-0.1$ (dashed lines).

region of the $\Delta \phi=\pi$ localized states. The associated snaking behavior is much more complex and a partial snaking branch (solid black curve) is shown in Fig. 3(b). When $\alpha<0$ the results are similar but not identical. The change of sign of $\alpha$ amounts to a translation of the forcing by half a period, and we use the label $\Delta \phi=0(\Delta \phi=\pi)$ to refer to solutions whose maxima (minima) are again in phase with the forcing maxima. The corresponding localized states are shown in Figs. 3(a) and 3(b) using dashed lines. As for $\alpha>0$, the resulting $\Delta \phi=0$ snaking branch is complete and resembles in all respects the $\phi=0$ snaking branch in $\mathrm{SH} 23$ with homogeneous forcing (Fig. 1). This is no longer the case for the $\Delta \phi=\pi$ localized states [Fig. 3(b)].

Figure 4 shows profiles of the localized solutions with $\Delta \phi=\pi$ for (a) $\alpha=0.1$ and (b) $\alpha=-0.1$ [Fig. 3(b)]. In both cases the snaking is incomplete: the $\alpha=0.1$ (resp. $\alpha=-0.1$ ) $\Delta \phi=\pi$ branch snakes normally until a 7- (resp. 8-) peak solution is reached but then doubles back and starts to snake towards states with a lower $L_{2}$ norm. As it does so, a defect is created that flattens the central region creating a state reminiscent of a two-pulse state [5,24]. The resulting behavior resembles that of localized structures in $\mathrm{SH} 23$ on nonperiodic domains with mixed boundary conditions $[25,26]$.

A reliable guide to the shift of the snaking regions for the $\Delta \phi=0$ and $\Delta \phi=\pi$ steady states is obtained by tracking the (a)

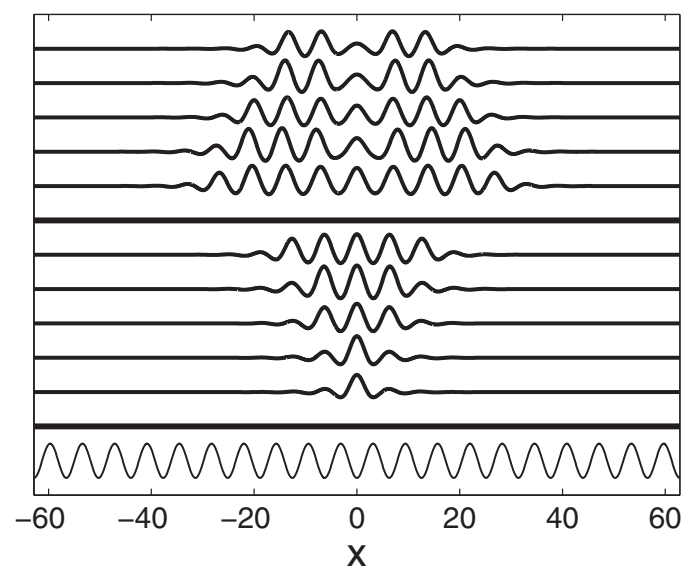

(b)

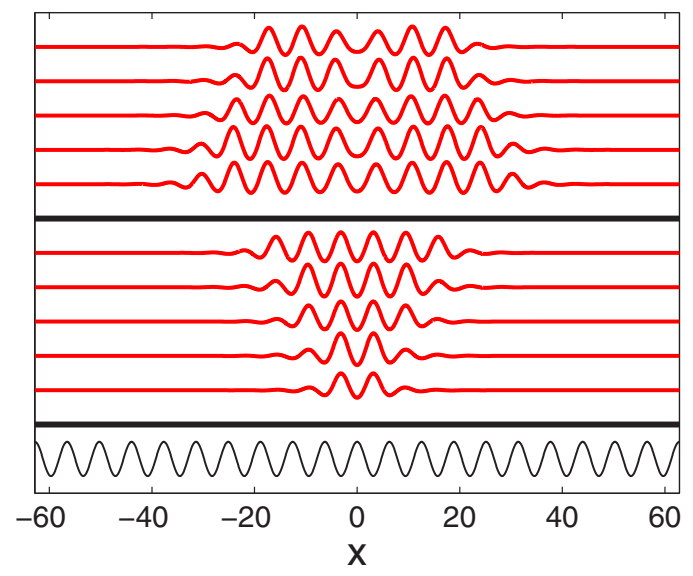

FIG. 4. (Color online) Solution profiles at successive saddle nodes along the $\Delta \phi=\pi$ branches from Fig. 3(b) for (a) $\alpha=0.1$ and (b) $\alpha=-0.1$, proceeding upward along the branch (profiles below the horizontal separation line) and then following it further as it starts to snake back down (profiles above the horizontal separation line). The bottom panel shows the forcing function $f_{p}$ (thin solid line).

motion of the saddle node of the periodic states and of the Maxwell point as a function of the amplitude $\alpha$ of the forcing $f_{p}$. Figure 5 shows the result. When $\alpha=0$ the saddle nodes of the $\Delta \phi=0, \pi$ periodic states coincide as do the corresponding Maxwell points, with the latter to the right of the former. As $|\alpha|$ increases both points move to the left for solutions of $\Delta \phi=0$ type (thick lines) but to the right for solutions of $\Delta \phi=\pi$ type (thin lines). Thus the snaking regions for the $\Delta \phi=0$ and $\Delta \phi=\pi$ solutions are pulled apart and ultimately do not overlap at all.

In order to appreciate more clearly the information that is represented in the above bifurcation diagrams we show in Fig. 6(a) the asymmetric rung states that accompany the above snaking branches. The figure shows a pair of such rungs for the parameter values of Fig. 3(a). These branches consist of stationary asymmetric states and connect an even branch consisting of solutions with minima at the symmetry point $x=0$ with even solutions with maxima at the symmetry point. As shown in Fig. 6(b), during this process the solution remains pinned at $x=0$; the transition takes place via the 


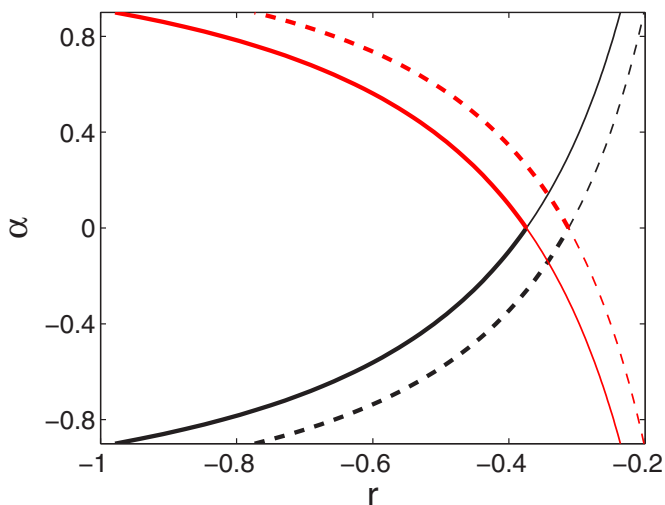

FIG. 5. (Color online) Location of the saddle nodes of the periodic branches (solid lines) and the associated Maxwell points (dashed lines) in the $(r, \alpha)$ plane for SH23. The thick (thin) lines represent $\Delta \phi=0(\Delta \phi=\pi)$ solutions.

gradual erosion of a peak at the right of the structure, resulting in an overall translation of the symmetry point from $x=0$ to $x=-\pi$. Similar transitions have been observed in integral models for neural fields where the rung states grow on one side while remaining pinned [27]. Such solutions can be translated

(a)

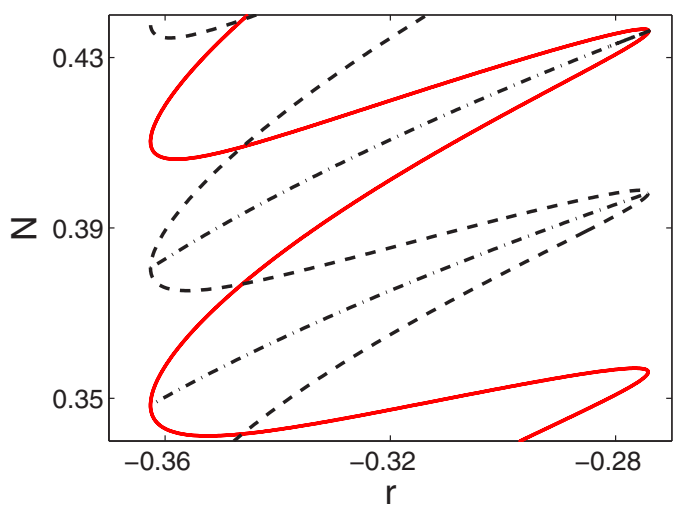

(b)

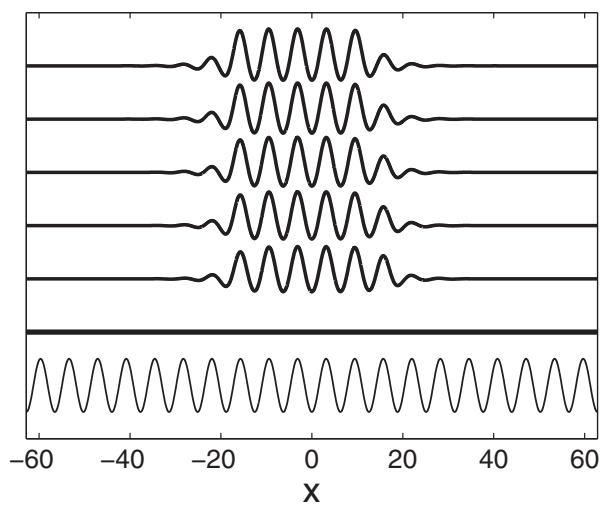

FIG. 6. (Color online) (a) Bifurcation diagrams for SH23 with the forcing $f_{p}$ and $\delta=1$, showing $N \equiv\|u\|_{L_{2}}$ as a function of $r$ for $\Delta \phi=0$ steady states. The forcing amplitude $\alpha=0.1$ (solid lines) and $\alpha=-0.1$ (dashed lines). Dash-dotted lines: rung states. (b) Sample solution profiles along the lower rung branch in (a) from left (bottom) to right (top). back to $x=0$ by translating the heterogeneity $f_{p}$ by $\pi$ to the right. Since the translation of $f_{p}$ by $\pi$ is equivalent to changing the sign of $\alpha$ it follows that such rung states connect symmetric states with opposite signs of the forcing amplitude $\alpha$ as indicated in Fig. 6(a). The resulting rungs are omitted from Fig. 3(a) for clarity.

The bifurcation diagrams obtained with cubic-quintic nonlinearity are shown in Figs. 7 and 8 for which we chose $\alpha= \pm 0.1$ and $\alpha= \pm 0.5$. As mentioned in Sec. II, there are two types of solutions: those with even parity with respect to the center $x=0$ (Fig. 7) and those with the odd parity with respect to the same point (Fig. 8). Note that we do not have to distinguish between even solutions with maxima that are in phase with the forcing $(\Delta \phi=0)$ and that are $\pi$ out of phase $(\Delta \phi=\pi)$ since these are related by the symmetry $(u, f) \rightarrow-(u, f)$ and likewise for odd solutions with positive (negative) slope at the location of maxima in the forcing $(\Delta \phi= \pm \pi / 2)$.

For even parity localized structures, the full snaking structure persists and the snaking region broadens as $|\alpha|$ increases. At the same time the two-limit snaking familiar from the homogeneous case turns into four-limit snaking: for $\alpha>0$ this leads to successive saddle nodes at locations $r_{1} \rightarrow r_{4} \rightarrow r_{2} \rightarrow r_{3} \rightarrow r_{1} \cdots$, while for $\alpha<0$ the sequence is $r_{2} \rightarrow r_{3} \rightarrow r_{1} \rightarrow r_{4} \rightarrow r_{2} \cdots$. Here $r_{i}<r_{j}$ whenever $i<j$, and $r_{1} \leqslant r \leqslant r_{4}$ represents the extent of the snaking region. Thus for $\alpha \neq 0$ every second saddle node on the left moves inwards relative to the other saddle nodes on the left and likewise for the saddle nodes on the right. However, with further increase in $|\alpha|$ the segments connecting $r_{2}$ and $r_{3}$ shrink, reaching zero length simultaneously throughout the whole structure at $|\alpha| \approx 0.23$, thereby restoring two-limit snaking, albeit with half the original snaking frequency [Fig. 7(b)]. The whole process is highly reminiscent of a similar sequence of transformations that takes place when the symmetry $u \rightarrow-u$ of SH35 is progressively broken [28,29]. The reason for this is the following. SH35, posed on $(0,2 \pi)$ with no forcing, has even solutions of the form $u(x)=\sum_{n} a_{n} \cos n x, n=$ $1,3,5, \ldots$ When a quadratic term $\alpha u^{2}$ is introduced, solutions take the form $u(x)=\sum_{n} a_{n} \cos n x, n=1,2,3, \ldots$, where $a_{2}=O(\alpha)$, etc. As a result changing $x$ to $x+\pi$ generates a distinct solution, and it is this fact that splits successive left (and right) saddle nodes in the snaking diagram. The introduction of the forcing $\alpha \cos x$ likewise changes solutions of SH35 of the form $u(x)=\sum_{n} a_{n} \cos n x, n=1,3,5, \ldots$ into $u(x)=\sum_{n} a_{n} \cos n x, n=1,2,3, \ldots$, where $a_{2}=O(\alpha)$, etc., and hence also splits successive left (and right) saddle nodes. The splitting is thus expected to be $O(\alpha)$, a conclusion confirmed in Sec. VI.

Figure 9 shows the initial development of the behavior just described as $|\alpha|$ increases from zero. The asymmetry between the $\alpha>0$ and $\alpha<0$ branches develops in the same way as in Ref. [28]. In both cases one ends up with four-limit snaking. Figure 10 reveals the consequences of this behavior for the rung states. When $\alpha=0$ these connect branches of even and odd states. When $\alpha \neq 0$ the even solutions split (recall that in-phase solutions with $\alpha<0$ correspond to out-of-phase solutions with $\alpha>0$ ) and the rung states deform into Z-shaped branches connecting even states to even states (Fig. 10). The solutions on the positive slope segment of the Z-shaped branch 
(a)

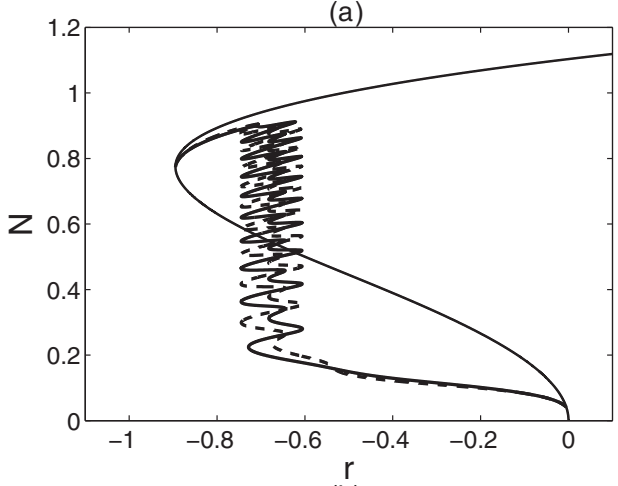

(b)

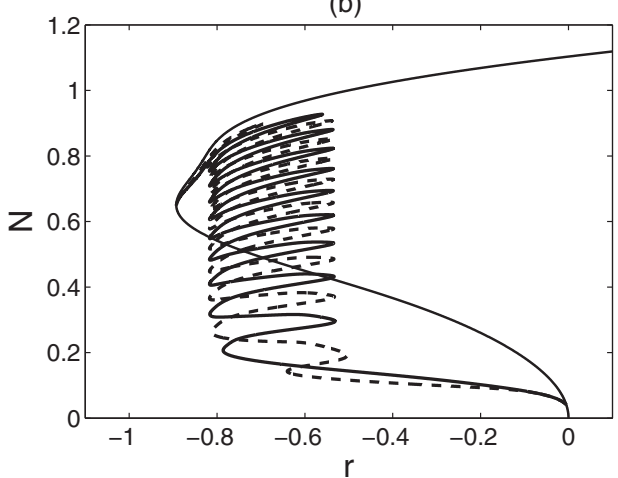

(c)

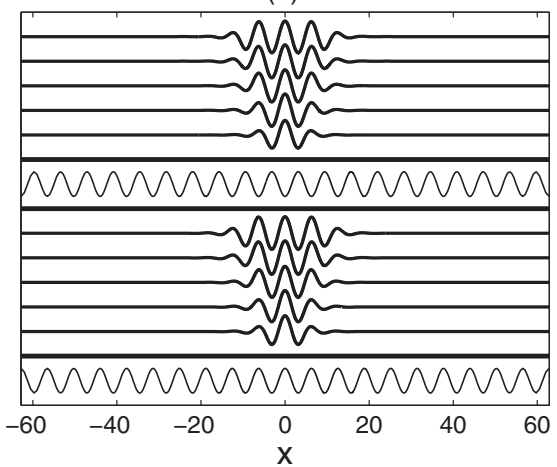

(d)

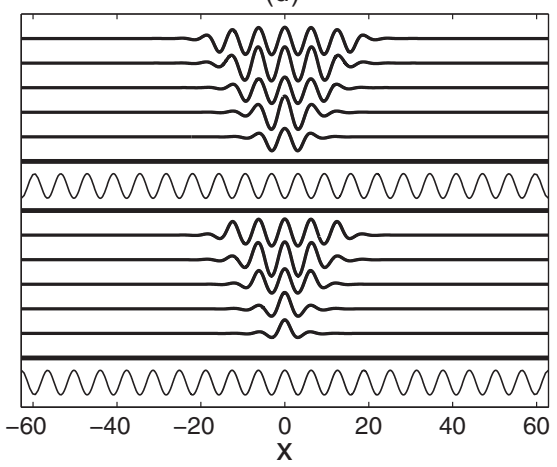

FIG. 7. Bifurcation diagrams and solution profiles for $\mathrm{SH} 35$ with the forcing $f_{p}$ and $\delta=1$, showing $N \equiv\|u\|_{L_{2}}$ as a function of $r$ for even parity branches when (a), (c) $\alpha= \pm 0.1$, (b), (d) $\alpha= \pm 0.5$. (a), (b) Branches of localized states for positive (negative) $\alpha$ are shown in solid (dashed) lines. (c), (d) Solution profiles at the first five saddle nodes along the snaking branches. Solutions with positive (negative) $\alpha$ are shown in the upper (lower) subpanels. The thin solid lines indicate the spatial profile $f_{p}$ of the heterogeneity. Because of the symmetry of SH35 with respect to $u \rightarrow-u$ profiles obtained by reflection in the horizontal axis are also solutions of SH35. (a)

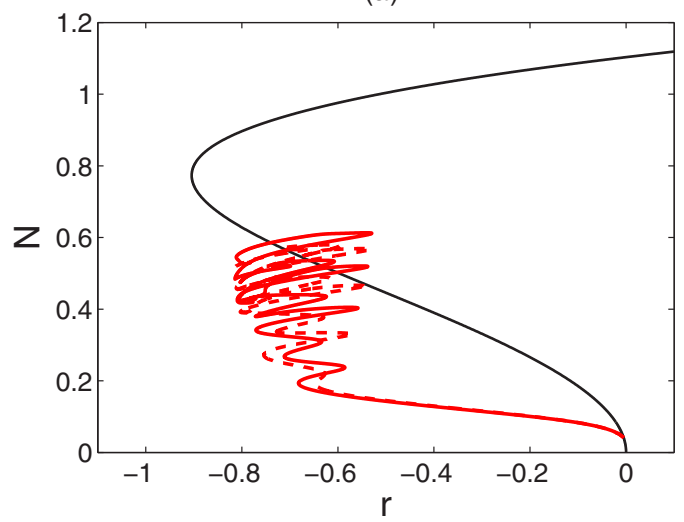

(b)

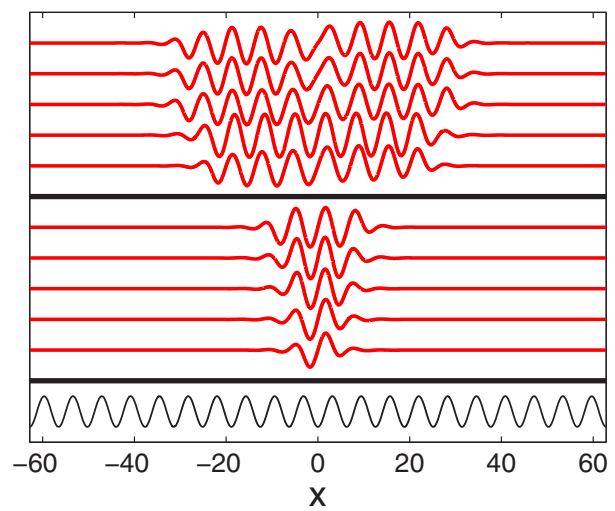

FIG. 8. (Color online) (a) Bifurcation diagram for SH35 with the forcing $f_{p}$ and $\delta=1, \alpha= \pm 0.5$ showing $N \equiv\|u\|_{L_{2}}$ as a function of $r$ for the odd parity branch. Localized branches of positive (negative) $\alpha$ are shown in solid (dashed) lines. (b) Solution profiles along the odd parity branch with $\alpha>0$ proceeding upward along the branch (profiles below the thick horizontal separation line) and then following it further as it starts to snake back down (profiles above the thick horizontal separation line). The thin solid line in the lowest panel indicates the spatial profile $f_{p}$ of the heterogeneity. Because of the symmetry of SH35 with respect to $u \rightarrow-u$ profiles obtained by reflection in the horizontal axis are also solutions.

follow a portion of the branch of odd parity states (for clarity omitted from the figure) and these are stable, just like the nearby odd parity states. With increasing $|\alpha|$ this stable portion of the asymmetric states is gradually eliminated and the branch stretches into a conventional rung, but now with half as many rungs as when $\alpha=0$, in a process that once again follows that identified in Ref. [28]. In view of this similarity we expect to find S-shaped branches of rung states as well. Such branches are indeed present, and Fig. 11 shows an example connecting the saddle nodes $r_{2}$ and $r_{3}$ on the $\alpha=0.1$ even parity branch. These solutions are all unstable; as $\alpha$ increases and the saddle nodes $r_{2}$ and $r_{3}$ annihilate the S-shaped rung branch shrinks to zero, again as in Ref. [28]. Figure 12 shows the corresponding rung states connecting odd parity states. In contrast to the rung states connecting even parity states these are unstable even along the positive slope segment of the branch. We emphasize that these states all originate in the asymmetric states at $\alpha=0$. As discussed in Sec. IV there are other asymmetric states as well. These are triggered by the translation eigenvalue and take 
(a)

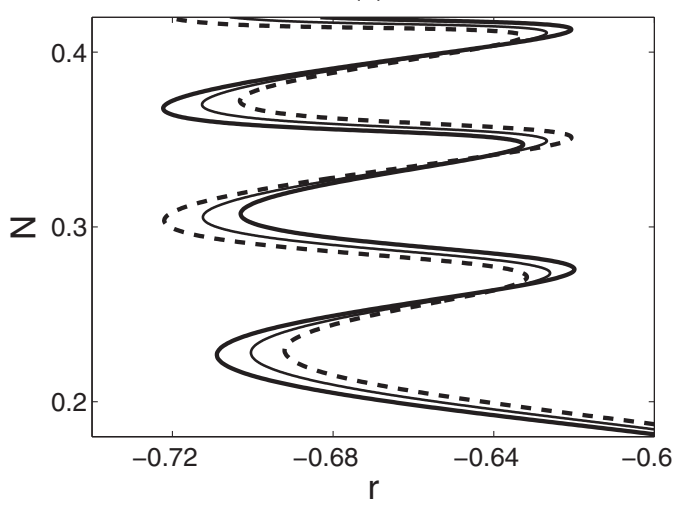

(b)

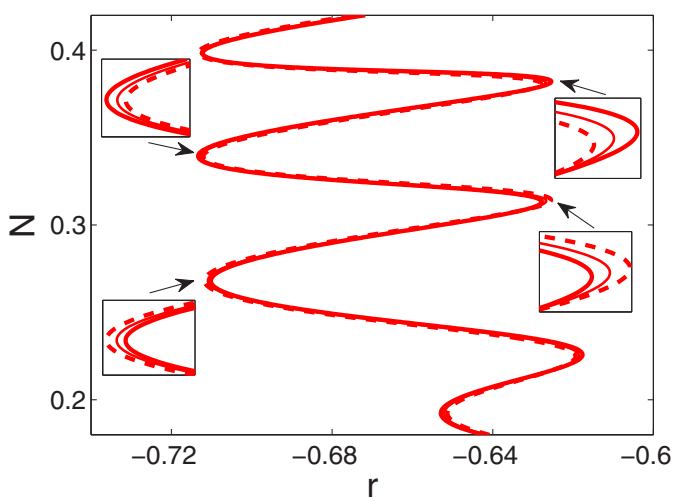

FIG. 9. (Color online) Bifurcation diagram for SH35 with the forcing $f_{p}, \delta=1$, showing $N \equiv\|u\|_{L_{2}}$ as a function of $r$ for (a) even parity solutions and (b) odd parity solutions. Thin line: $\alpha=0$. Thick solid (dashed) line: $\alpha=0.03(-0.03)$.

the form of a body mode in which the asymmetry is distributed across the whole structure instead of being confined to the fronts at either side of the structure.

For odd parity states, the bifurcation behavior [Fig. 8(a)] is qualitatively similar to that of the $\Delta \phi=\pi$ solutions in $\mathrm{SH} 23$. The full snaking structure exists for small $|\alpha|$ and collapses earlier and earlier as $|\alpha|$ increases, but the sign of $\alpha$ has a much smaller effect on the bifurcation behavior than for the even states. Solution profiles at the bottom five saddle nodes along the snaking branch are shown in Fig. 8(b). Figure 13 shows the location of the saddle nodes of the periodic branches and the Maxwell points as functions of the forcing amplitude $\alpha$. This dependence on $\alpha$ in SH35 differs from that in SH23. The curves in Fig. 13 are again symmetric with respect to the axis $\alpha=0$, which is a consequence of the $T_{\pi} R_{2}$ symmetry of the periodic states, i.e., symmetry with respect to up-down reflection followed by a translation in $x$ by half a wavelength. The bistable region for the odd parity periodic branch broadens as $|\alpha|$ increases, but the branch is affected much less by the periodic forcing than the even parity branch. For the latter, the bistability region first narrows as $|\alpha|$ increases from zero but then rapidly broadens once $|\alpha|$ exceeds a certain threshold. In both cases the corresponding Maxwell points follow in step.

It is important to observe that the $\mathrm{Z}$ and $\mathrm{S}$ branches have a different physical origin. The $\mathrm{Z}$ branches are generated via

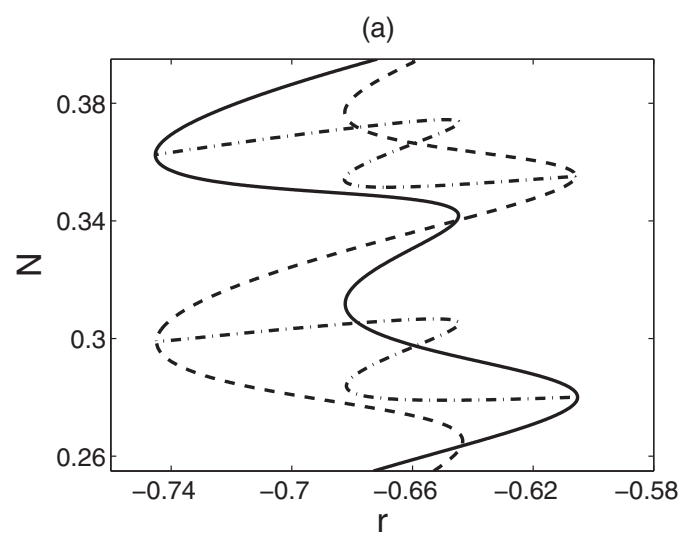

(b)

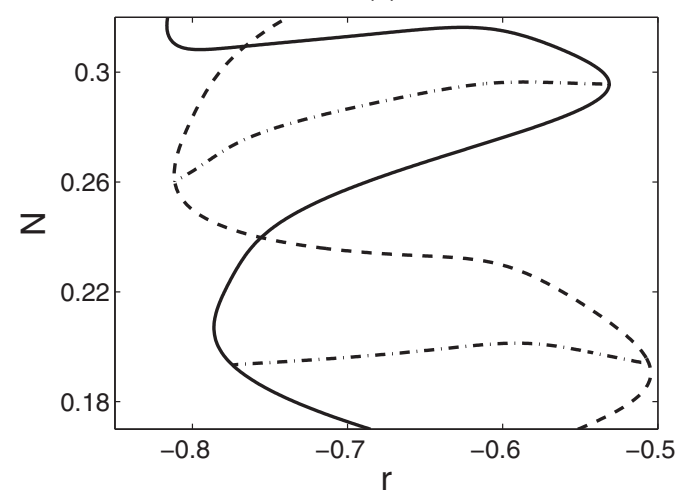

FIG. 10. Bifurcation diagrams for SH35 with the forcing $f_{p}$ and $\delta=1$, showing $N \equiv\|u\|_{L_{2}}$ as a function of $r$ for even parity steady states [cf. Fig. 9(a)] and the corresponding rung states (dashed-dotted lines). The forcing amplitude (a) $\alpha= \pm 0.1$ and (b) $\alpha= \pm 0.5$. The rung states are the result of destabilization of the phase mode.

a phase instability localized at the location of the fronts at the front and back of the corresponding localized structure. Figure 14 shows the eigenfunctions at the endpoints of the Z-shaped branches shown in Fig. 10(a). For comparison Fig. 15 shows the corresponding eigenfunctions at the endpoints of

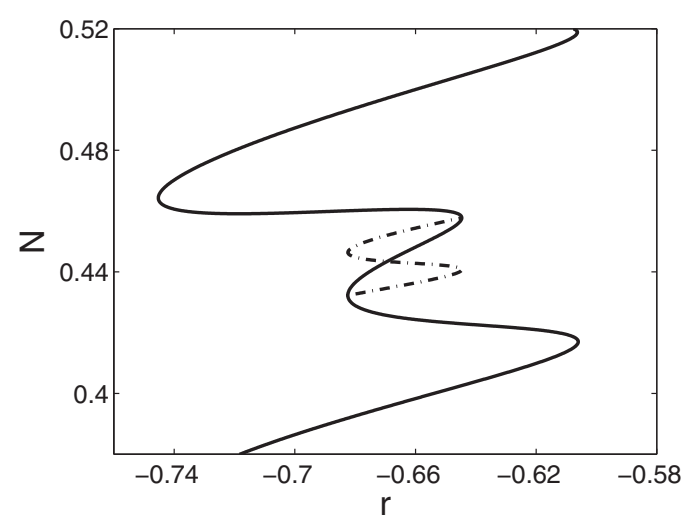

FIG. 11. Bifurcation diagram for SH35 with the forcing $f_{p}$ and $\delta=1$, showing $N \equiv\|u\|_{L_{2}}$ as a function of $r$ for even parity steady states [cf. Fig. 9(a)] and the corresponding rung states (dashed-dotted lines). The forcing amplitude $\alpha=0.1$. The $\mathrm{S}$-shaped branch of rung states is the result of destabilization of the translation mode. 


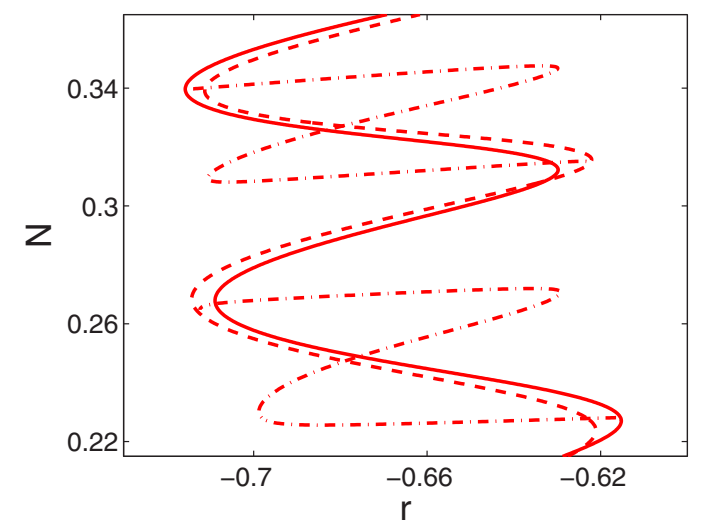

FIG. 12. (Color online) Bifurcation diagram for SH35 with the forcing $f_{p}$ and $\delta=1$, showing $N \equiv\|u\|_{L_{2}}$ as a function of $r$ for odd parity steady states [cf. Fig. 9(b)] and the corresponding rung states (dashed-dotted lines). The forcing amplitude $\alpha= \pm 0.1$.

the S-shaped branches shown in Fig. 11. In contrast to the phase eigenfunctions the latter extend across the whole localized structure. In Sec. IV we show that states of this type are generated by the destabilization of the translation mode by the applied forcing $f_{p}$. The above discussion indicates that as $\alpha$ increases the phase instability comes to dominate while the translation or drift instability gradually disappears.

\section{B. Bump heterogeneity $f_{b}$}

We now describe the results obtained when the heterogeneity $f_{b}(x)$ with $\sigma=\pi / 2$ is applied. This heterogeneity is strongly localized and consequently outside a region of length of the order of one natural wavelength of the pattern the solutions resemble those of the homogeneous system. Bifurcation diagrams obtained with $\alpha= \pm 0.5$ are shown in Fig. 16 for SH23 and Fig. 17 for SH35. As soon as the bump is imposed, the equation loses both the continuous and discrete translation invariance. As a result periodic solutions are no longer present. Instead, spatially localized solutions

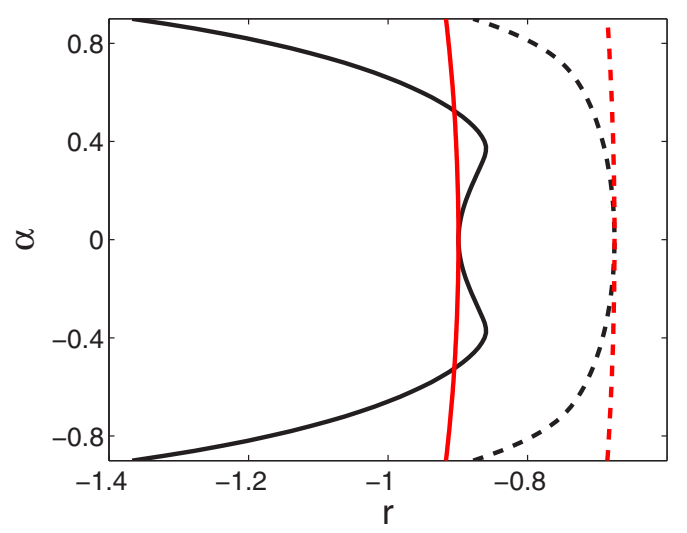

FIG. 13. (Color online) Location of the saddle node of the periodic branches (solid lines) and the Maxwell points (dashed lines) in the $(r, \alpha)$ plane for SH35. The black [red (or gray)] lines correspond to even (odd) parity states. (a)

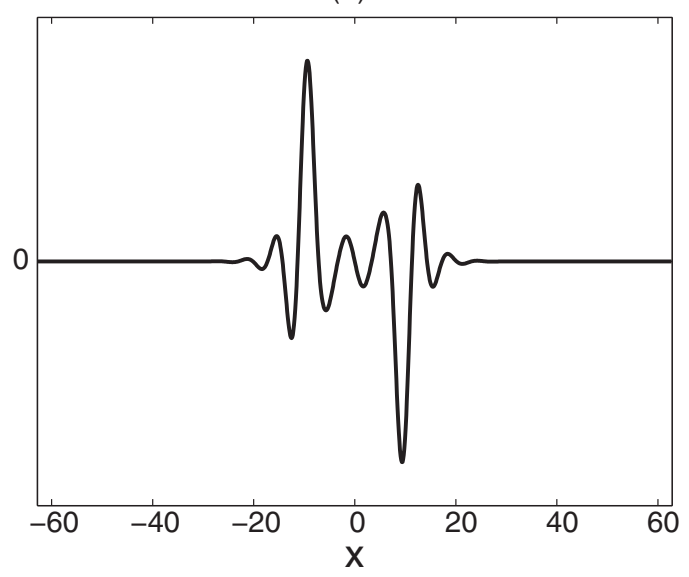

(b)

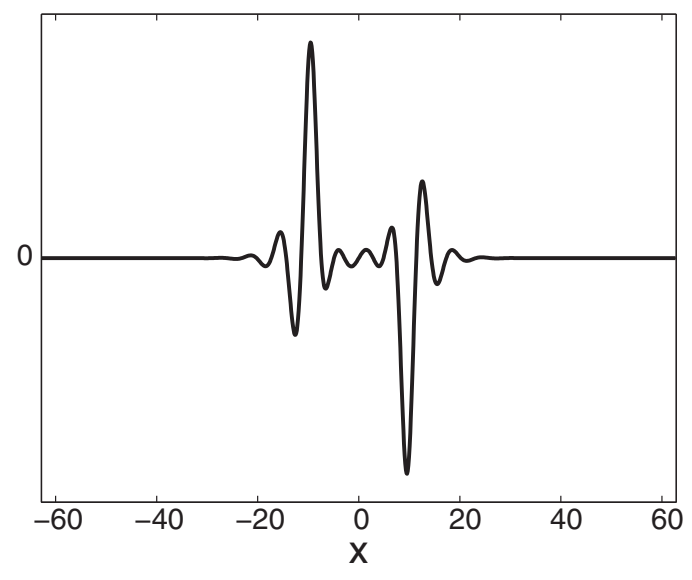

FIG. 14. Eigenfunctions at (a) the left end and (b) the right end of the upper Z-shaped rung branch shown in Fig. 10(a). Both eigenfunctions are localized at the fronts at either end of the localized structure indicating that the Z-shaped branch is created as a result of the destabilization of the $\alpha=0$ phase mode.

are created in a primary bifurcation as described in Ref. [25] for a system where like symmetries are destroyed through the imposition of mixed (or Robin) boundary conditions. Depending on the sign of $\alpha$, two types of localized solutions may be produced. The localized solutions emerge at the location where forcing is maximum. Thus when $\alpha$ is positive (negative) the forcing is weakened (strengthened) at the center of the domain and the localized solution emerges from the edge (center) of the domain as shown in Figs. 16(c), 16(d), 17(c), and 17(d) in the upper (lower) subpanels at the bottom. In each case a pair of branches of localized solutions results, in phase and out of phase with the forcing, and each exhibits snaking. When $\alpha>0$ both the in-phase and out-of-phase solutions exhibit regular snaking [solid lines in Figs. 16(a) and 16(b)]. In contrast, when $\alpha<0$ the branches depart at the bottom from the classical picture [dashed lines in Figs. 16(a) and 16(b)]. This results from the fact that the localized states now coincide with the imposed bump, so that the localized structures sense the associated rapid variation of the forcing parameter, resulting in a prominent shift of the first left saddle node towards lower values of $r$. However, this change in behavior impacts only the first few saddle nodes, and further up 
(a)

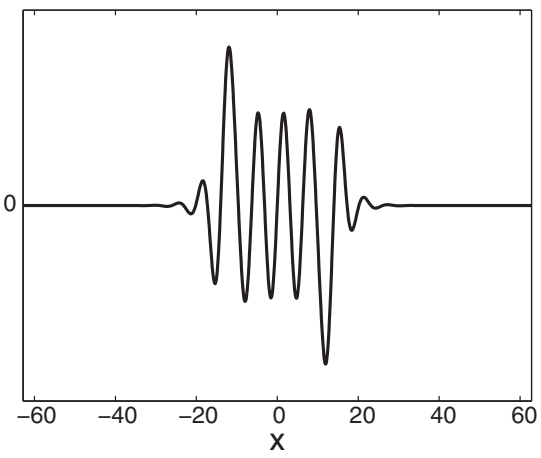

(b)

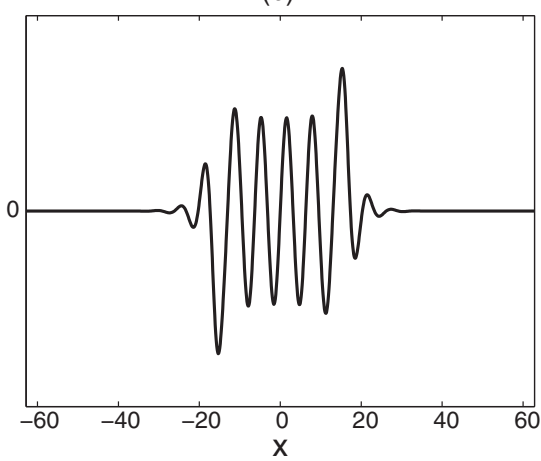

FIG. 15. Eigenfunctions at (a) the lower end and (b) the upper end of the S-shaped rung branch shown in Fig. 11. Both eigenfunctions are spatially extended indicating that the $\mathrm{S}$-shaped branch is created as a result of the destabilization of the $\alpha=0$ translation mode.

the snaking diagram the $\alpha<0$ branch falls rapidly into phase with the $\alpha>0$ branch. This is, of course, a consequence of the fact that once the structure is broader than a few wavelengths the effect of the heterogeneity is overwhelmed by nucleation of additional oscillations in the homogeneous part of the forcing. The resulting branches do not terminate when the domain is full but instead undergo a prominent overshoot required to accommodate the defect before turning continuously towards larger $r$ and producing large amplitude spatially periodic structures with a defect at the location of the bump. This is so for $\alpha>0$ as well [Figs. 16(a) and 16(b)]. Once again the resulting behavior resembles closely that familiar from systems exhibiting localized states with non-Neumann boundary conditions $[25,30]$.

Figures 17(a) and 17(b) show the corresponding results for SH35, again for $\alpha= \pm 0.5$. Except for the doubled frequency of the snaking branches the results resemble qualitatively those in Figs. 16(a) and 16(b) for SH23. We have not carried out a detailed study of the asymmetric states with the bump heterogeneity. However, in Figs. 18 and 19 we show sample bifurcation diagrams showing rung states for $\mathrm{SH} 23$ and $\mathrm{SH} 35$, respectively. In contrast to Figs. 6 and 10 these connect branches of $\Delta \phi=0$ states to themselves, and not to the $\Delta \phi=\pi$ states. We believe that this is a consequence of the spatial localization of the forcing function $f_{b}$. This pushes the $\Delta \phi=0$ and $\Delta \phi=\pi$ states a distance $\Gamma / 2$ apart, in contrast to their separation $\lambda / 2$ when the forcing function is $f_{p}$ (Sec. III A). (a)

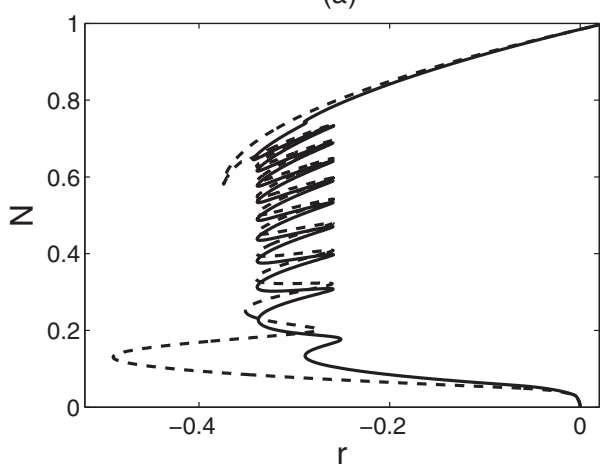

(b)

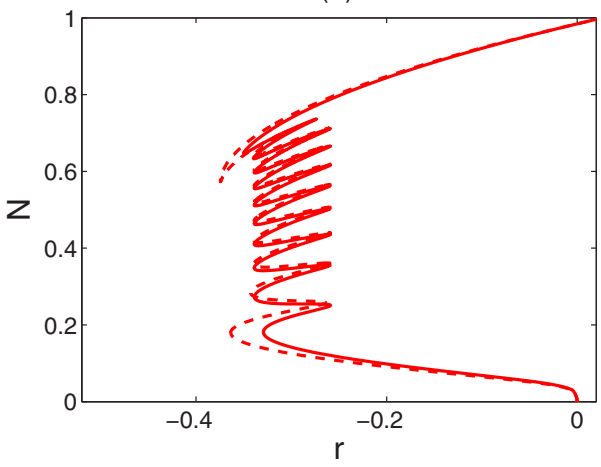

(c)

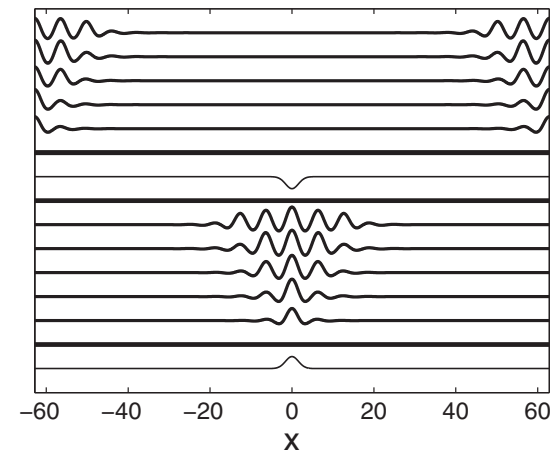

(d)

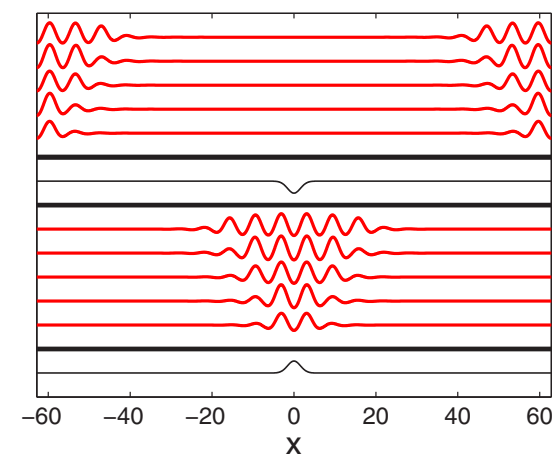

FIG. 16. (Color online) (a), (b) Bifurcation diagrams showing $N \equiv\|u\|_{L^{2}}$ as a function of $r$ for SH23 with the forcing $f_{b}$ and $\alpha= \pm 0.5$. Solution branches with $\Delta \phi=0$ (resp. $\pi$ ) are shown in (a) [resp. (b)]. Localized branches for positive (negative) $\alpha$ are shown in solid (dashed) lines. (c), (d) Solution profiles at the first five saddle nodes along the snaking branches. The profiles for positive (negative) $\alpha$ are shown in the upper (resp. lower) subpanels. The thin solid line at the bottom of each subpanel indicates the spatial profile of the heterogeneity. 
(a)

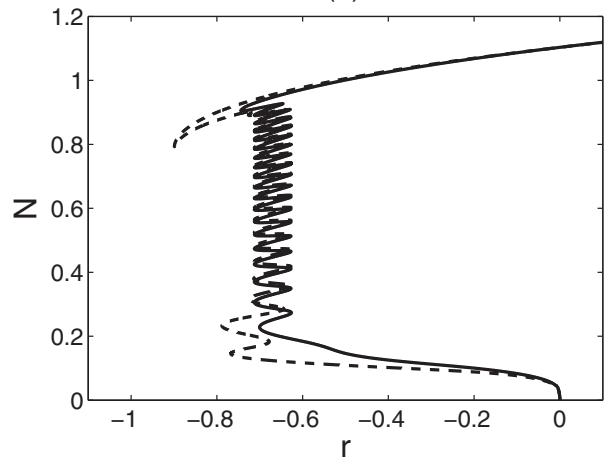

(b)

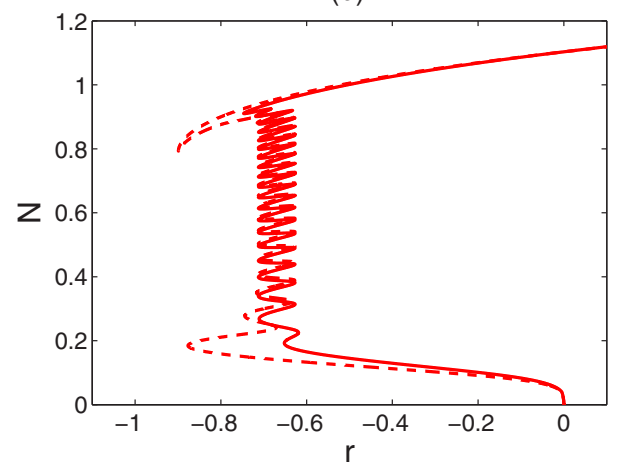

(c)

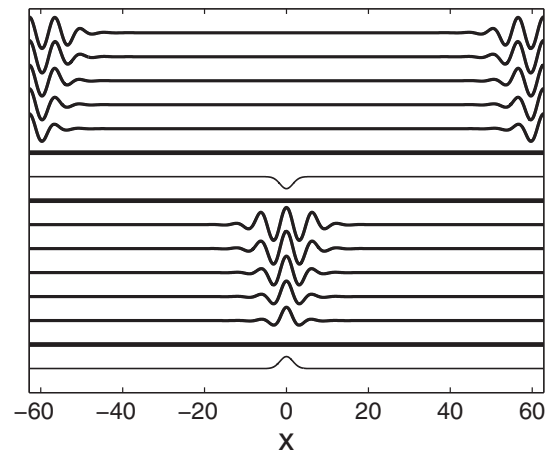

(d)

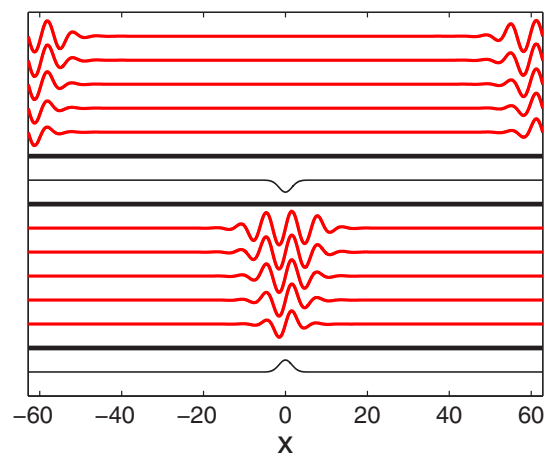

FIG. 17. (Color online) (a), (b) Bifurcation diagrams showing $N \equiv\|u\|_{L^{2}}$ as a function of $r$ for SH35 with the forcing $f_{b}$ and $\alpha= \pm 0.5$. Solution branches with even (resp. odd) parity are shown in (a) [resp. (b)]. Localized branches for positive (negative) $\alpha$ are shown in solid (dashed) lines. (c), (d) Solution profiles at the first five saddle nodes along the snaking branches. The profiles for positive (negative) $\alpha$ are shown in the upper (resp. lower) subpanels. The thin solid line at the bottom of each subpanel indicates the spatial profile of the heterogeneity. (a)

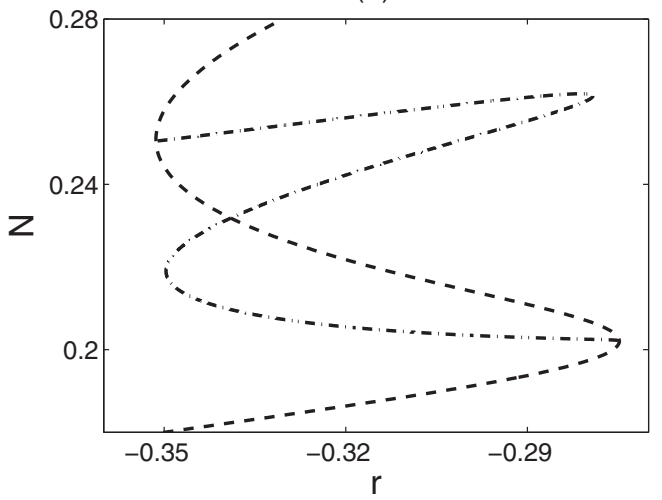

(b)

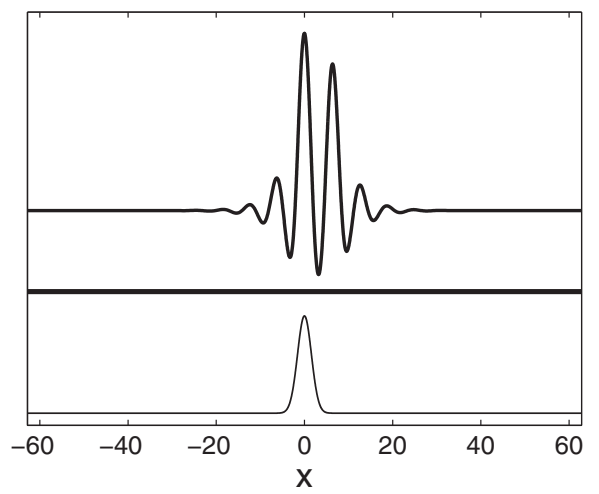

FIG. 18. (a) Bifurcation diagrams showing $N \equiv\|u\|_{L^{2}}$ as a function of $r$ for SH23 with the forcing $f_{b}$ and $\alpha=-0.5$. Dashed line: even parity $\Delta \phi=0$ localized states. Dashed-dotted line: asymmetric rung states. (b) A stable asymmetric state with $r=-0.307$ and $N=0.248$ on the middle part of the rung [reached in Fig. 26(a)]. The bump profile is shown in the lowest panel.

\section{STABILITY}

So far we have not discussed the stability properties of the solutions we have found. These are complicated by the presence of the non-neutral translation eigenvalue. This eigenvalue is responsible for the drift of the different steady states with $\alpha=0$ as soon as the forcing $f$ is turned on, as further discussed in Sec. VII. This drift also selects stationary states from the one-parameter family of such states present when $\alpha=0$. It is these "remaining" stationary states that are represented in the bifurcation diagrams of Secs. III A and III B.

The drift velocity can be obtained by an asymptotic calculation when the magnitude of the heterogeneity $|\alpha|$ is small, i.e., $\alpha=\epsilon \alpha_{1}$ with $\alpha_{1}=O(1)$ and $\epsilon \ll 1$. Introducing the long time scale $T \equiv \epsilon t$ we write the solution in the form

$$
u(x, t)=u_{0}\left(x-x_{c}\right)+\epsilon u_{r}(x, T),
$$

where $u_{0}$ is a stable stationary solution obtained for $\alpha=0$, $x_{c} \equiv x_{c}(T)$ is the location of a reference point on the solution and $u_{r}$ is a correction. Substituting these relations into Eq. (1) we obtain

$-\epsilon u_{0}^{\prime} \dot{x}_{c}=\epsilon r \alpha_{1} f u_{0}+\epsilon\left[r-\left(1+\partial_{x x}\right)^{2}+N^{\prime}\left(u_{0}\right)\right] u_{r}+O\left(\epsilon^{2}\right)$. 
(a)

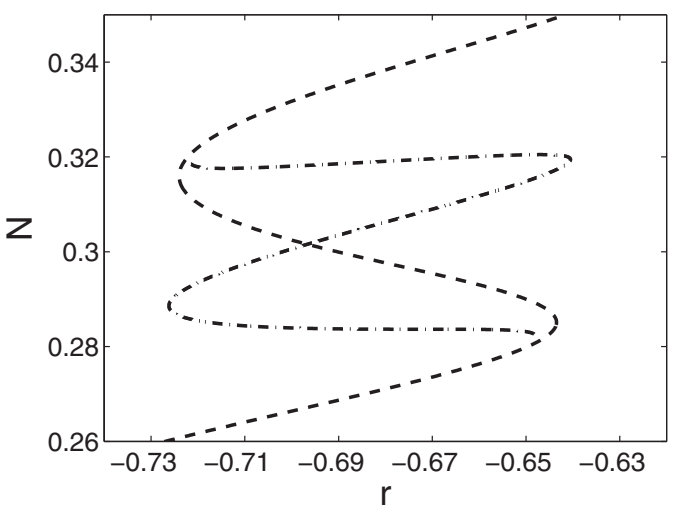

(b)

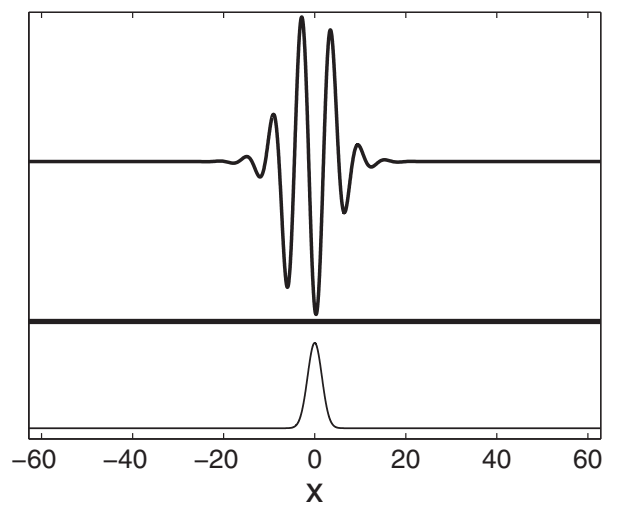

FIG. 19. (a) Bifurcation diagrams showing $N \equiv\|u\|_{L^{2}}$ as a function of $r$ for SH35 with the forcing $f_{b}$ and $\alpha=-0.5$. Dashed line: odd parity localized states. Dashed-dotted line: asymmetric rung states. (b) A stable asymmetric state with $r=-0.686$ and $N=0.305$ on the middle part of the rung [reached in Fig. 26(c)]. The bump profile is shown in the lowest panel.

The contribution of the correction term $u_{r}$ can be eliminated by multiplying Eq. (10) by $u_{0}^{\prime}$ and integrating over the domain, yielding

$$
\dot{x}_{c}\left\langle u_{0}^{\prime 2}\right\rangle=-r \alpha_{1}\left\langle f u_{0} u_{0}^{\prime}\right\rangle+O(\epsilon)=\frac{r \alpha_{1}}{2}\left\langle f^{\prime} u_{0}^{2}\right\rangle+O(\epsilon) .
$$

Here $\langle\cdots\rangle$ denotes integration over the domain. From this relation we obtain the drift velocity

$$
\frac{d x_{c}}{d t}=\frac{r \alpha}{2} \frac{\left\langle f^{\prime} u_{0}^{2}\right\rangle}{\left\langle u_{0}^{\prime 2}\right\rangle}+O\left(\epsilon^{2}\right) .
$$

It follows that stationary solutions correspond to the zeros of $\left\langle f^{\prime} u_{0}^{2}\right\rangle$. There are two types of such solutions, those for which the maxima of the solution coincide with the maxima of $f$ $(\Delta \phi=0)$, and those for which the minima coincide with the maxima of $f(\Delta \phi=\pi)$.

We may use these results to determine the stability of these stationary states with respect to the translation mode for $|\alpha| \ll 1$. We suppose that the stationary solution at $t=0$ is shifted from its equilibrium position by a small spatial displacement $\Delta x$. We have

$$
\begin{aligned}
u_{t}(x, 0) & =\left.\partial_{t} u_{0}\left(x-x_{c}(t)\right)\right|_{t=0}+\text { h.o.t. } \\
& =-u_{0}^{\prime}(x-\Delta x) \frac{r \alpha}{2} \frac{\left\langle f^{\prime}(x) u_{0}(x-\Delta x)^{2}\right\rangle}{\left\langle u_{0}^{\prime 2}\right\rangle}+\text { h.o.t. } \\
& =u_{0}^{\prime}(x) r \alpha \Delta x \frac{\left\langle f^{\prime}(x) u_{0}(x) u_{0}^{\prime}(x)\right\rangle}{\left\langle u_{0}^{\prime 2}\right\rangle}+\text { h.o.t. }
\end{aligned}
$$

To obtain this result we have repeatedly used the fact that at $\alpha=0$ the quantity $\left\langle f^{\prime} u_{0}^{2}\right\rangle=0$. From Eq. (13), we now obtain the eigenvalue of the translation mode,

$$
\lambda \approx \frac{r \alpha}{2} \frac{\left\langle f^{\prime \prime}(x) u_{0}(x)^{2}\right\rangle}{\left\langle u_{0}^{\prime 2}\right\rangle},
$$

indicating that the translation eigenvalue depends linearly on $\alpha$ when $|\alpha| \ll 1$. We emphasize that the corresponding eigenfunction is a body mode and so is nonzero across the whole structure. This is in contrast with the symmetrybreaking phase modes familiar from the $\alpha=0$ case which are wall modes: for these modes the eigenfunction is nonzero only at the location of the fronts. Of course, when a steady solution loses stability and $\lambda$ becomes positive the instability does not result in a steadily drifting state; instead the instability creates a pair of steady but asymmetric states towards which the solution evolves [31]. For the body mode the tilt of the resulting asymmetric state is distributed throughout the structure; for a wall mode the asymmetry manifests itself at the location of the fronts only. Examples of such evolution are shown in Sec. VII.

Computations show that for the forcing $f=f_{p}$ the eigenvalue $\lambda<0$ everywhere along the $\Delta \phi=0$ branch in SH23, while $\lambda>0$ everywhere along the $\Delta \phi=\pi$ branch (Fig. 20), regardless of the sign of $\alpha$. Thus the translation mode selects the states along the positive slope segments of the $\Delta \phi=0$ branch, cf. Ref. [22], but does not generate any additional bifurcations along the snaking branches. The situation is not nearly so simple for SH35 as discussed next.

Figure 21 shows the corresponding results for SH35. In contrast to Fig. 20 we see that the translation eigenvalue oscillates about $\lambda=0$ as one proceeds up both the even and odd parity branches, thereby repeatedly triggering and suppressing the drift instability. To appreciate the impact of the associated drift instability we show in Fig. 22(a) the five leading eigenvalues $\lambda$ of the linear problem describing the stability of the localized states in SH35, plotted as a function of the norm $N$ of the solution. The four eigenvalues visible in Fig. 22(a) are obtained by continuation from the unforced $(\alpha=0)$ problem, and all correspond to wall modes. The eigenvalue $\lambda>0, N \ll 1$, represents the amplitude eigenvalue. It oscillates repeatedly through zero, each zero corresponding to a saddle node of $u(x)$. The eigenvalue $\lambda<0$, $N \ll 1$, represents the phase eigenvalue responsible for the generation of the asymmetric rung states. This eigenvalue also oscillates with $N$, and its oscillations approach exponentially rapidly the oscillations in the amplitude eigenvalue so that high up the snaking branch the rung states bifurcate essentially from the saddle nodes of the branch $[18,22]$. This is due to the fact that both instabilities are associated with instabilities of the fronts that connect the structure to the background 

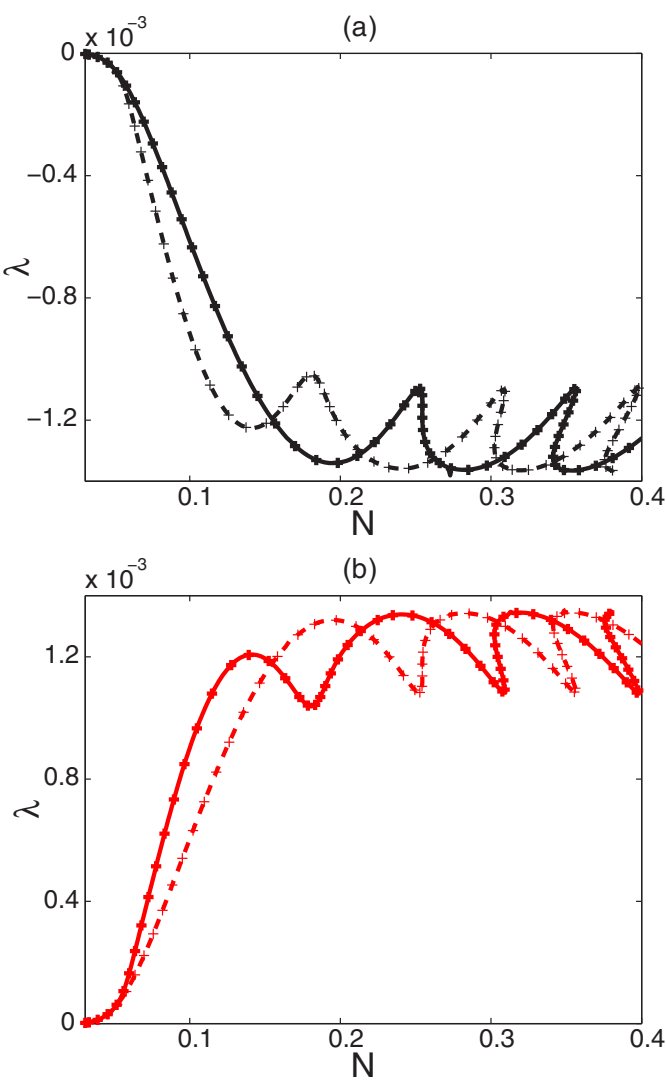

FIG. 20. (Color online) The translation eigenvalue of (a) $\Delta \phi=0$ and (b) $\Delta \phi=\pi$ localized states in SH23 with the forcing $f_{p}$ and $\delta=1$ as a function of $N \equiv\|u\|_{L_{2}}$. Solid (dashed) line: $\alpha=10^{-2}$ $\left(\alpha=-10^{-2}\right)$. + : Prediction from Eq. (14).

homogeneous state; for large structures the instabilities at the front and back are essentially independent, and the amplitude and phase modes therefore become degenerate $[18,20]$. The next two eigenvalues are always negative, emphasizing the fact that the subsequent eigenvalues do not trigger instabilities. The remaining fifth eigenvalue is almost invisible in Fig. 22(a) and is therefore enlarged in Fig. 22(b). This eigenvalue is the translation eigenvalue and is nonzero only because $\alpha \neq 0$. We see that high up the snaking branch this eigenvalue crosses zero at every second saddle node, implying that half of the amplitude-stable segments is drift-stable and half is drift-unstable. This is so for both $\alpha>0$ and $\alpha<0$. Explicit calculation shows that in SH35 the oscillation of the translation mode destabilizes the branch segments connecting the saddle nodes at $r_{2}$ and $r_{3}$ regardless of the sign of $\alpha$ while the segments connecting $r_{1}$ and $r_{4}$ remain stable. This is in contrast to Ref. [28], where the segments $r_{2}<r<r_{3}$ also correspond to stable even parity states. Figure 21 shows that for small $\alpha$ the analytical prediction (14) of the translation eigenvalue agrees very well with the exact eigenvalue determined numerically. However, for larger $\alpha$ the exact eigenvalue moves gradually downward, ultimately resulting in the pairwise disappearance of all neutral drift modes. These locations in the parameter $\alpha$ correspond, of course, to the annihilation of the saddle nodes $r_{2}$ and $r_{3}$ and the disappearance of the associated S-shaped rung branch, as described in Sec. III A.
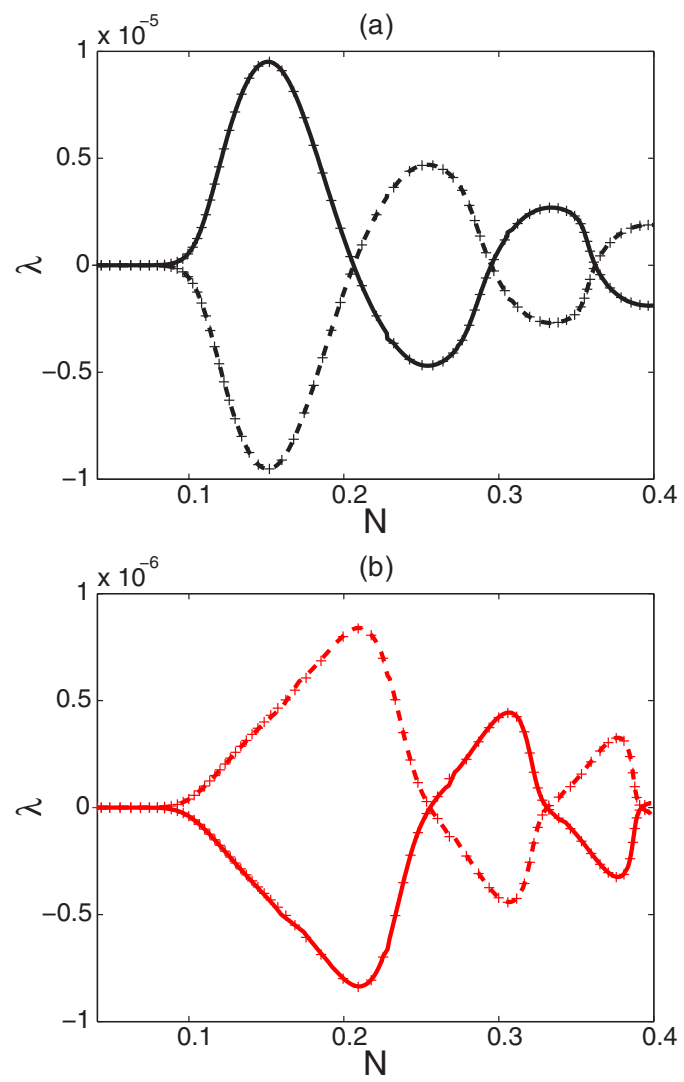

FIG. 21. (Color online) The translation eigenvalue of (a) even and (b) odd localized states in SH35 with the forcing $f_{p}$ and $\delta=1$ as a function of $N \equiv\|u\|_{L_{2}}$. Solid (dashed) line: $\alpha=10^{-4}\left(\alpha=-10^{-4}\right)$. + : Prediction from Eq. (14).

In contrast, the odd states are gradually destabilized as $|\alpha|$ becomes nonzero. This is a consequence of the dramatically different behavior of the phase and translation eigenvalues of the odd parity states. These modes are both even in $x$ and consequently reconnect near every crossing point, i.e., when the phase eigenvalue is near $\lambda=0$, resulting in avoided crossings of the type seen in Fig. 23(b), cf. Ref. [32]. The figure shows the phase eigenvalue increasing rapidly from zero along the branch of odd parity states together with a more slowly increasing (negative) translation eigenvalue. Both these eigenvalues reach $O(1)$ values before returning to the vicinity of $\lambda=0$. In this region the phase eigenfunction temporarily broadens to resemble the translation eigenfunction. Once the phase eigenvalue departs from the vicinity of $\lambda=0$ the eigenfunction becomes confined near the front and back of the structure as in the $\alpha=0$ case. The converse is true for the translation mode. As the translation eigenfunction departs from $\lambda=0$ the eigenfunction becomes more and more confined to the fronts at either end of the structure. Thus the phase and translation modes repeatedly mix as one proceeds up the branch of odd parity states. In contrast, for the parameter values in Fig. 22 the phase and translation eigenvalues of the even parity states do not mix because they do not intersect near the crossing points $\lambda=0$. As a result both eigenvalues repeatedly cross $\lambda=0$, triggering instabilities that evolve into the rung states shown in Fig. 10. In contrast, the very brief 
(a)
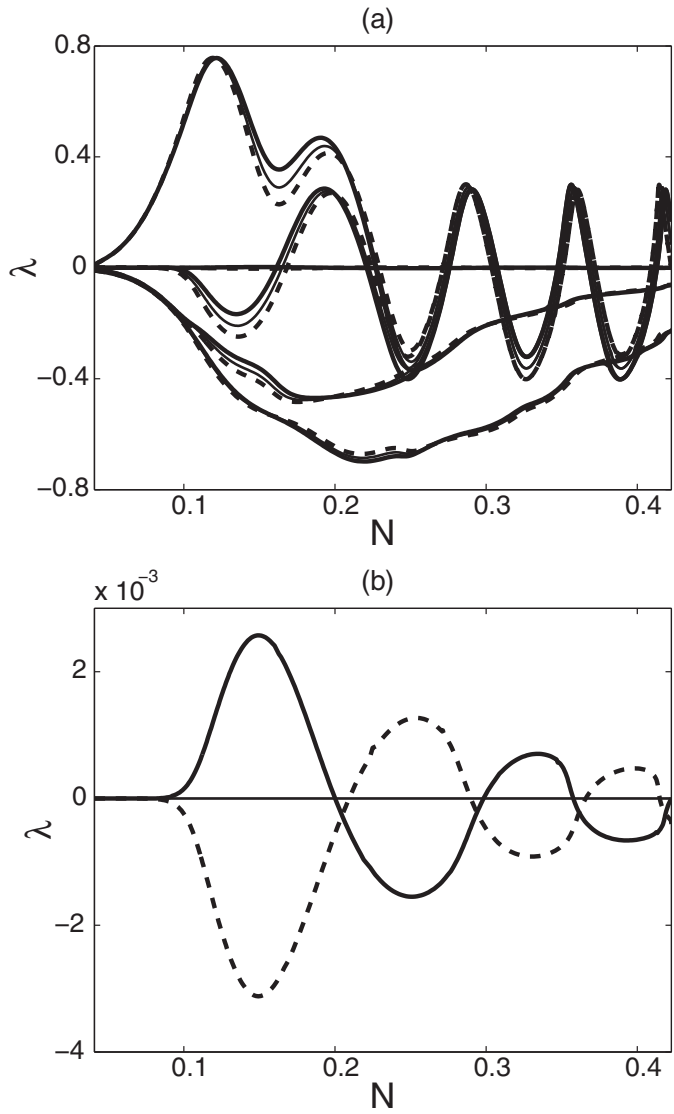

FIG. 22. The linear stability of even localized states in SH35 with the forcing $f_{p}$ and $\delta=1$. (a) The first five leading eigenvalues of the even parity stationary solutions in Fig. 9(a) as functions of $N \equiv\|u\|_{L_{2}}$, and (b) enlargement of the translation eigenvalue. The thin lines correspond to $\alpha=0$, while the thick solid (dashed) lines correspond to $\alpha=0.03(\alpha=-0.03)$.

crossings of $\lambda=0$ in the odd parity case only produce short segments of asymmetric states such as the S-shaped branch shown in Fig. 11.

The stability properties of localized states with the forcing $f_{b}$ are similar but not identical. This time we find that in $\mathrm{SH} 23$ both $\Delta \phi=0$ and $\Delta \phi=\pi$ states are stable along the positive slope branch segments when $\alpha>0$ while only the $\Delta \phi=0$ states are stable along the positive slope branch segments when $\alpha<0$. The stability of the $\Delta \phi=\pi$ states when $\alpha>0$ is a consequence of the fact that these states are localized away from the bump and hence inherit the stability properties of the $\alpha=0$ system. Similarly, both even and odd states of SH35 are stable along the positive slope branches when $\alpha>0$, while only the even states are stable along these segments when $\alpha<0$; the odd states are all unstable.

\section{HETEROGENEOUS FORCING ON A $O(\Gamma)$ SPATIAL SCALE}

In this section, we present the results when the underlying forcing has a much larger, $O(\Gamma)$, spatial scale, where we recall $\Gamma$ is the domain size. The only heterogeneity considered here is $f_{p}$ and we choose $\delta=0.05$, thereby generating a forcing with a single wavelength in the spatial domain. The forcing (a)

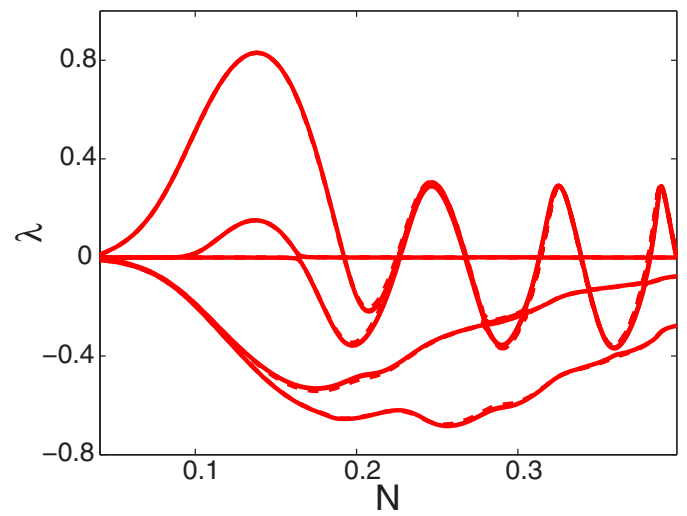

(b)

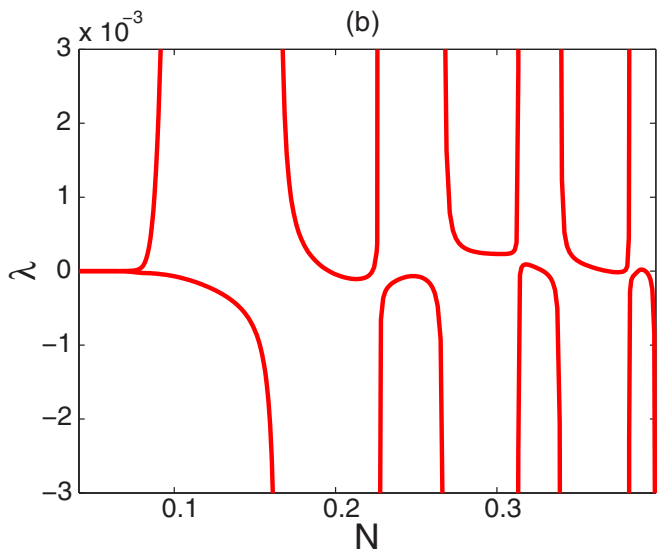

FIG. 23. (Color online) The linear stability of odd localized states in SH35 with the forcing $f_{p}$ and $\delta=1$. (a) The first five leading eigenvalues of the odd parity stationary solutions in Fig. 9(b) as functions of $N \equiv\|u\|_{L_{2}}$, and (b) enlargement of the translation (lower curve) and phase (upper curve) eigenvalues when $\alpha=0.03$. In (a) the thin lines correspond to $\alpha=0$, while the thick solid (dashed) lines correspond to $\alpha=0.03(\alpha=-0.03)$.

resulting from this $f_{p}$ satisfies the symmetry $R_{2} T_{\Gamma / 2}$; as a result the bifurcation branches for $\alpha$ positive and negative coincide. The bifurcation diagrams obtained for $\mathrm{SH} 23$ and $\mathrm{SH} 35$ are qualitatively similar and are shown in Fig. 24. The symmetries of the problem are the same as for the bump heterogeneity $f_{b}$ and translation invariance is therefore completely broken. Thus localized structures are again created in a primary bifurcation. These solutions localize rapidly as their norm increases, in the same way as for the bump heterogeneity, and thereafter undergo snaking until the domain is filled. The branches evolve into a large amplitude domain-filling state with further increase in $r$. However, instead of undergoing snaking in a well-defined interval in $r$ as in the previous section, this time the snaking structure exhibits a prominent slant with a slope that decreases rapidly as $|\alpha|$ increases. Once again the stability of the solutions is similar to that for the case $\alpha=0$, with solutions on the positive slope segments stable and the remainder unstable.

As the branch departs from the bifurcation point at $r=0$, the solution amplitude builds up at the location where the forcing is the largest, yielding localized oscillations: for $\alpha>0$ the oscillations therefore first appear at the boundary of the 
(a)

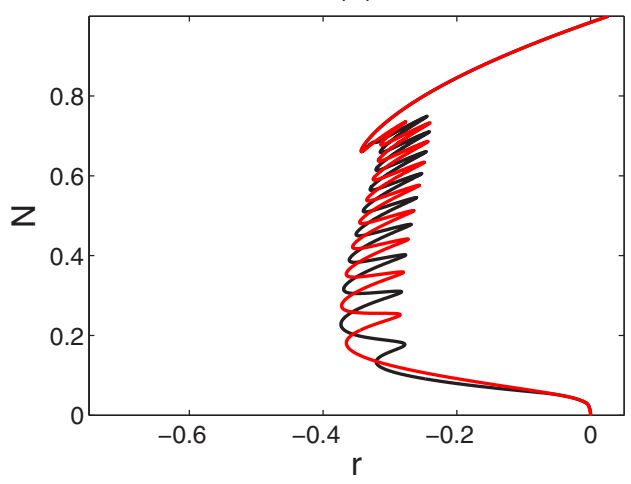

(b)

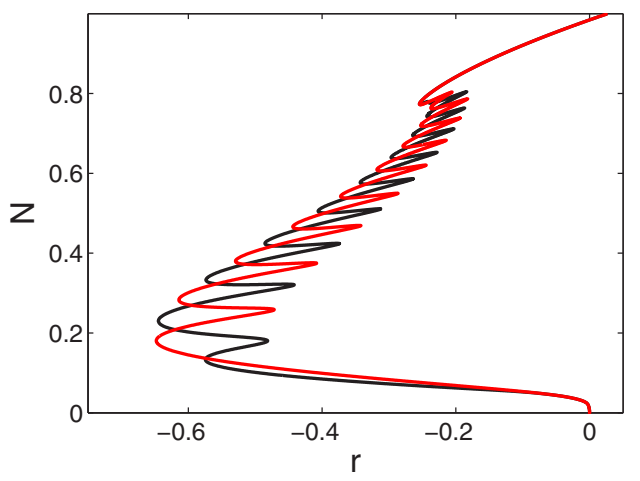

(c)

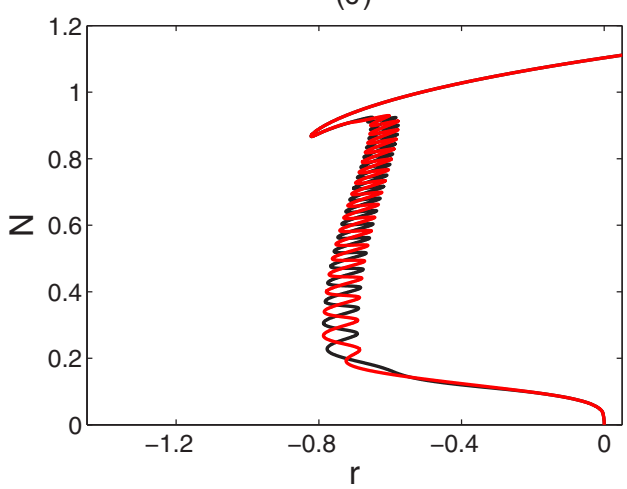

(d)

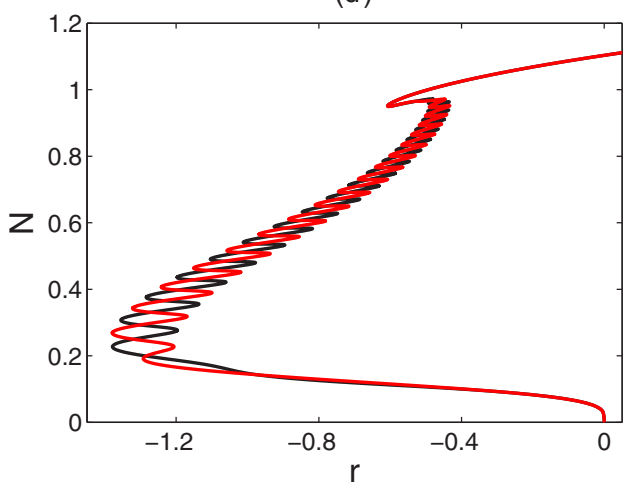

FIG. 24. (Color online) Bifurcation diagrams showing $N \equiv$ $\|u\|_{L_{2}}$ as a function of $r$ for (a), (b) SH23 and (c), (d) SH35 with the forcing $f_{p}$ and $\delta=0.05$. Panels (a) and (c) correspond to $\alpha= \pm 0.1$, while (b) and (d) are for $\alpha= \pm 0.5$. In (a) and (b) the black [red (or gray)] snaking branch corresponds to solutions with $\phi=0(\phi=\pi)$; in (c) and (d) the black [red (or gray)] lines correspond to even (odd) parity states.

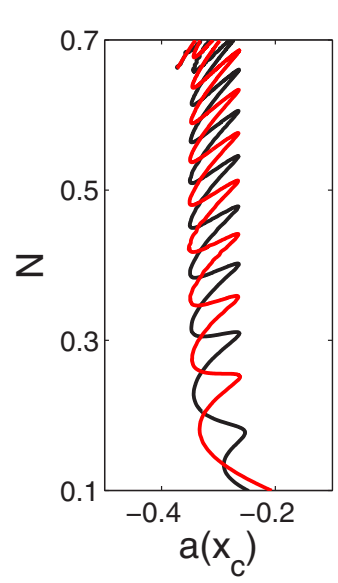

(a)
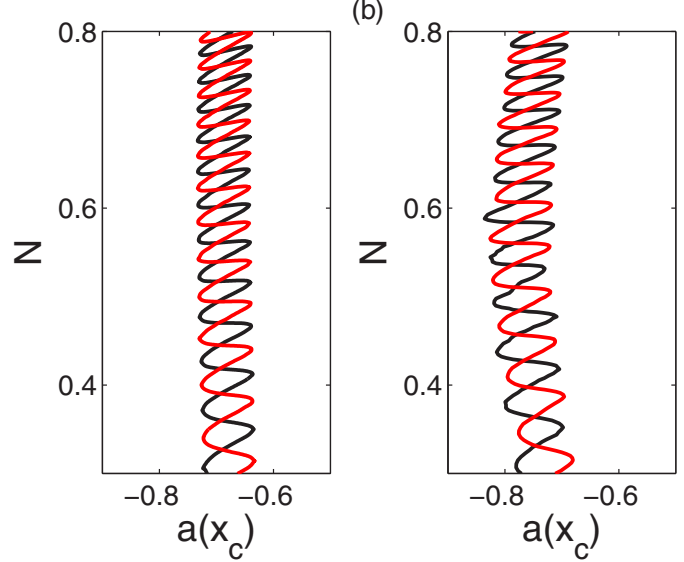

FIG. 25. (Color online) Bifurcation diagrams showing $N \equiv$ $\|u\|_{L_{2}}$ from Fig. 24 as a function of $a_{c} \equiv r\left[1+\alpha \cos \left(x_{c}\right)\right]$, for (a) SH23 and (b) SH35 with the heterogeneity $f_{p}$ and $\delta=0.05$. Left panels correspond to $\alpha= \pm 0.1$, while the right panels correspond to $\alpha= \pm 0.5$.

domain while for $\alpha<0$ they do so at the center. However, as already explained the resulting branches coincide. During the snaking regime the solution nucleates new oscillations at locations with progressively weaker forcing, thereby leading to a tilt of the snaking structure that increases with increasing $|\alpha|$. Unlike the situation with $\delta=1$, in the present case there is a substantial difference in scales between the intrinsic spatial scale of the Swift-Hohenberg equation (set equal to $2 \pi$ ) and the slow spatial scale on which the forcing varies (set equal to $\Gamma$ ). As a result the forcing is locally homogeneous but offset by an amount depending on the spatial phase of the heterogeneity. To confirm this hypothesis, we replot the snaking structure with the forcing parameter $r$ replaced by the effective forcing parameter $a_{c} \equiv r\left[1+\alpha \cos \left(\delta x_{c}\right)\right]$, where $x_{c}$ represents the spatial location of the front that connects the rolls to the zero state. This location is determined numerically by first locating the extremal points and then applying a cubic spline to determine the shape of the envelope between them. The quantity $x_{c}$ is defined as the location of the intersection between $|u|=0.1$ and the envelope curve. Figure 25 shows the snaking structure in a bifurcation diagram with $r$ replaced by $a_{c}$. In this representation the localized states are almost exactly vertically aligned, as in standard snaking, and the 
snaking interval is almost independent of $\alpha$, at least for small $|\alpha|$, indicating that structures such as that shown in Fig. 24 resembling slanted snaking are indeed the result of a slow variation of the effective forcing parameter, much as in systems with a conserved quantity when these are defined on a periodic domain with a finite period [6,33]. For larger values of $|\alpha|$ the vertical alignment is less good, an observation we attribute to the fact that as $N$ and hence $r$ increases the front profile also changes; as a result the front location $x_{c}$, as constructed above, does in fact depend weakly on $r$, and this dependence is expected to grow with increasing $|\alpha|$.

\section{SPLITTING OF THE SADDLE NODES}

To investigate the splitting of the saddle nodes of the periodic states with $\Delta \phi=0, \pi$ as $\alpha$ becomes nonzero, we use the approach of Ref. [29] to calculate the change in the location of the saddle node $r_{s n}$. We assume that the solutions along a particular branch can be parameterized by a parameter $s$ and pick $s \equiv\|u\|_{L_{2}}^{2}$ to simplify the calculation. This parametrization is valid at least locally around the saddle nodes. Equation (1) in the stationary case can thus be written as

$$
F[u(x ; \alpha, s), r(\alpha, s) ; \alpha]=0,
$$

where $u$ and $r$ now depend on the parameters $\alpha$ and $s$. Differentiation of $F$ with respect to $\alpha$ and $s$ gives

$$
\begin{gathered}
L u_{\alpha}+F_{r} r_{\alpha}+F_{\alpha}=0, \\
L u_{s}+F_{r} r_{s}=0,
\end{gathered}
$$

where

$$
L \equiv a-\left(1+\partial_{x x}\right)^{2}+N_{u}
$$

is a self-adjoint linear operator. Since $u_{s}$ is the marginal mode at the saddle-node bifurcation, $L u_{s}$ vanishes at the saddle node. Equation (17) shows that this condition translates into the statement that $r_{s}=0$ at the saddle node. Multiplying Eq. (16) by $2 u_{s}$ and integrating the result over the domain using the fact that $L$ is self-adjoint, we find that for $\alpha=0$

$$
2\left(\left\langle u_{s} F_{r}\right\rangle r_{\alpha}+\left\langle u_{s} F_{\alpha}\right\rangle\right)=r_{\alpha}+r\left\langle f u^{2}\right\rangle_{s}=0
$$

at the saddle node. Here $\langle v\rangle \equiv \int_{-\Gamma / 2}^{\Gamma / 2} v(x) d x$. When $\alpha \neq 0$ the saddle node is located at $r_{s n}=r(\alpha, s(\alpha))$, where $s=s(\alpha)$ is determined by the condition $r_{s}=0$. We have $r_{s n}^{\prime}=r_{\alpha}+r_{s} s^{\prime}$ and $r_{s}=0$ at the saddle node. Thus the splitting of $r_{s n}$ up to $O(\alpha)$ takes the form

$$
r_{s n}(\alpha)=r_{s n}(0)\left(1-\alpha\left\langle f u^{2}\right\rangle_{s}\right)+O\left(\alpha^{2}\right) .
$$

Relation (20) can be used to explain the observed splitting of the saddle nodes of the periodic branches in the numerical continuation results given in earlier sections. Owing to the nonzero mean of the periodic solutions in $\mathrm{SH} 23$, the quantity $\left\langle f_{p} u^{2}\right\rangle$ is positive (resp. negative) for the periodic branch with $\Delta \phi=0$ (resp. $\Delta \phi=\pi$ ). The solutions grow in amplitude as one passes through the saddle nodes implying that $\left\langle f_{p} u^{2}\right\rangle_{s}$ has the same sign as $\left\langle f_{p} u^{2}\right\rangle$. The relation (20) now implies that the $\Delta \phi=0$ branch has $r_{s n}(\alpha)<r_{s n}(0)$ while the $\Delta \phi=\pi$ branch has $r_{s n}(\alpha)>r_{s n}(0)$ in agreement with the numerical results. A similar argument can be used to explain the shift in saddle nodes of the snaking structures observed in SH23 and $\mathrm{SH} 35$. The interaction between the forcing and the nucleation of new oscillations at the front and back of the structures which fall into regions of reduced forcing generates the shifts in the saddle nodes observed in Figs. 24(a) and 24(b).

However, for $\mathrm{SH} 35$ with the heterogeneity $f_{p},\left\langle f u^{2}\right\rangle$ vanishes for both even and odd periodic solutions, and the calculation to $O(|\alpha|)$ is insufficient to determine the splitting of the saddle node. A calculation up to $O\left(\alpha^{2}\right)$ is required. The second derivatives of $F$ with respect to $\alpha$ and $s$ are

$$
\begin{gathered}
L u_{\alpha \alpha}+2\left(L_{\alpha}+r_{\alpha} L_{r}\right) u_{\alpha}+N_{u u} u_{\alpha}^{2}+F_{r r} r_{\alpha}^{2} \\
\quad+F_{r} r_{\alpha \alpha}+F_{\alpha \alpha}+2 F_{r \alpha} r_{\alpha}=0, \\
L u_{\alpha s}+r_{s} L_{r} u_{\alpha}+\left(r_{\alpha} L_{r}+L_{\alpha}\right) u_{s}+N_{u u} u_{\alpha} u_{s} \\
\quad+F_{r r} r_{\alpha} r_{s}+F_{r} r_{\alpha s}+F_{\alpha r} r_{s}=0, \\
L u_{s s}+2 r_{s} L_{r} u_{s}+N_{u u} u_{s}^{2}+F_{r r} r_{s}^{2}+F_{r} r_{s s}=0 .
\end{gathered}
$$

From Eq. (1), we see that $F_{\alpha \alpha}$ and $F_{r r}$ are both zero when $\alpha=0$. Multiplying Eqs. (21)-(23) by $2 u_{s}$ and integrating the result using the fact that $L$ is self-adjoint, the following relations are obtained at the saddle node when $\alpha=0$ :

$$
r_{\alpha \alpha}=-2\left\langle\left(N_{u u} u_{\alpha}+2 r f-2 r\left\langle f u^{2}\right\rangle_{s}\right) u_{\alpha} u_{s}\right\rangle+2 r\left(\left\langle f u^{2}\right\rangle_{s}\right)^{2},
$$

$$
\begin{aligned}
& r_{\alpha s}=-2\left\langle\left(N_{u u} u_{\alpha}+r f-r\left\langle f u^{2}\right\rangle_{s}\right) u_{s}^{2}\right\rangle, \\
& r_{s s}=-2\left\langle u_{s}^{3} N_{u u}\right\rangle .
\end{aligned}
$$

The second derivative of $r_{s n}$ takes the form

$$
r_{s n}^{\prime \prime}=r_{\alpha \alpha}+2 r_{\alpha s} s^{\prime}+r_{s} s^{\prime \prime}+r_{s s}\left(s^{\prime}\right)^{2}=r_{\alpha \alpha}-\frac{r_{\alpha s}^{2}}{r_{s s}},
$$

where we made use of $r_{s}=0$ and $s^{\prime}=-r_{\alpha s} / r_{s s}$ to obtain the last equality. The splitting to $O\left(\alpha^{2}\right)$ therefore takes the form

$$
\begin{aligned}
r_{s n}(\alpha)= & r_{s n}(0)\left(1-\alpha\left\langle f u^{2}\right\rangle_{s}\right) \\
& +\alpha^{2}\left[\left\langle\left(N_{u u} u_{\alpha}+r f-r\left\langle f u^{2}\right\rangle_{s}\right) u_{s}^{2}\right\rangle^{2} /\left\langle u_{s}^{3} N_{u u}\right\rangle\right. \\
& \left.-\left\langle\left(N_{u u} u_{\alpha}+2 r f-2 r\left\langle f u^{2}\right\rangle_{s}\right) u_{\alpha} u_{s}\right\rangle+r\left(\left\langle f u^{2}\right\rangle_{s}\right)^{2}\right] \\
& +O\left(|\alpha|^{3}\right) .
\end{aligned}
$$

The factor of $O\left(\alpha^{2}\right)$ in Eq. (28) can be determined numerically, but $u_{\alpha}$ needs to be solved for from Eq. (16) before evaluating the integrals. The operator $L$ has two marginal modes at the saddle node, $u_{s}$ and $u_{x}$, leading to the conditions

$$
\left\langle u u_{\alpha}\right\rangle=0, \quad\left\langle u_{x} u_{\alpha}\right\rangle=0 .
$$

The first of these ensures the $L^{2}$-norm of the solution is fixed when $s$ is not changed while the second fixes the center of the solution at $x=0$. The calculation shows good agreement with the results obtained from numerical continuation of the periodic state. For SH35, we obtain $r_{s n}^{\prime \prime}(0) \approx 0.89954\left(r_{s n}^{\prime \prime}(0) \approx\right.$ -0.04263 ) for the even (odd) branch, while the corresponding values obtained from Eq. $(28)$ are $r_{s n}^{\prime \prime}(0) \approx 0.90054\left(r_{s n}^{\prime \prime}(0) \approx\right.$ $-0.04263)$. The calculation can be extended to arbitrarily high order and we expect that the nonmonotonic behavior of the 
saddle nodes with increasing $|\alpha|$ observed in Fig. 13 can be captured within such a higher order calculation.

For the localized states in $\mathrm{SH} 35\left\langle f_{p} u^{2}\right\rangle$ and $\left\langle f_{p} u^{2}\right\rangle_{s}$ are both nonzero and the generic results apply. However, their magnitude for the even state is approximately 10 times that for the odd states, thereby explaining the much smaller saddlenode splitting in Fig. 9(b) than in Fig. 9(a).

\section{PATTERN DYNAMICS IN THE PRESENCE OF HETEROGENEOUS FORCING}

The presence of spatially dependent forcing has significant effects on pattern selection. The results in Sec. IV indicate that

(a)

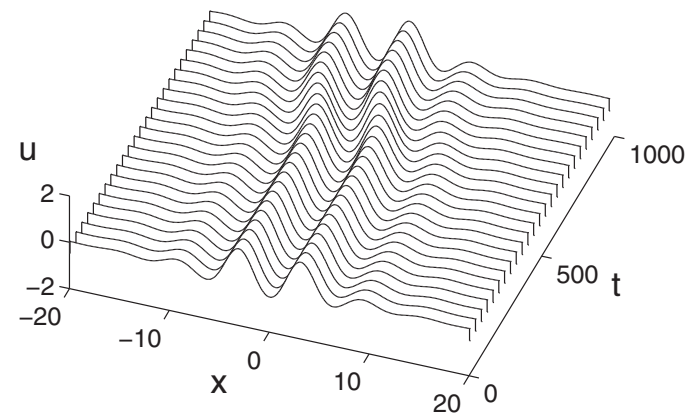

(b)

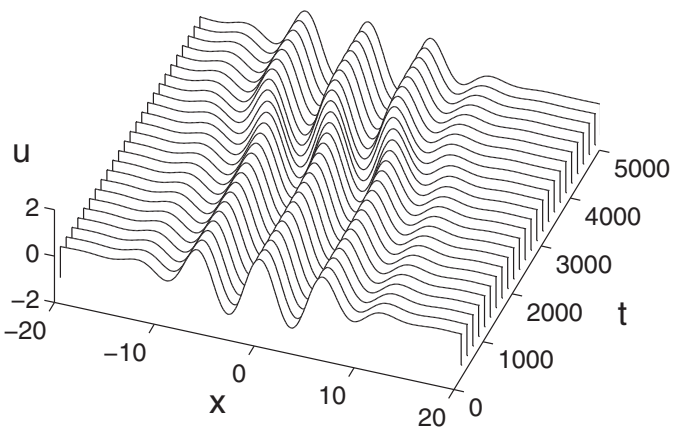

(c)

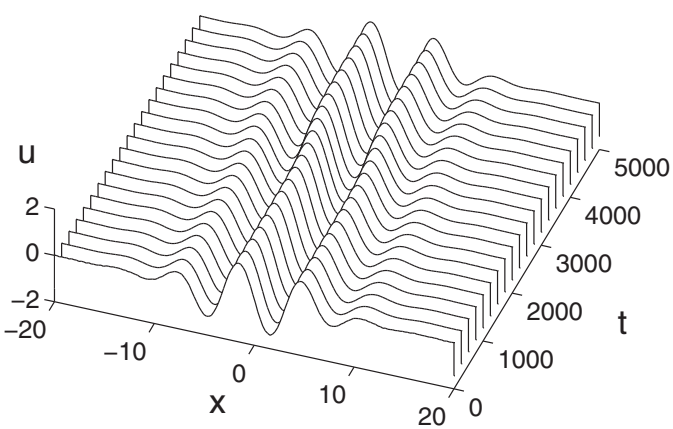

FIG. 26. Time evolution of (a) an unstable $\Delta \phi=\pi$ localized solution in SH23 with forcing $f_{b}$ and $\alpha=-0.5$, (b) an unstable even localized solution in SH35 with forcing $f_{p}$ with $\alpha=0.1, \delta=1$, and (c) an unstable odd localized solution in SH35 with forcing $f_{b}$ with $\alpha=-0.5$. in SH23 the $\Delta \phi=\pi$ states that are stable under homogeneous forcing become unstable in the presence of the forcing $f_{p}$ with nonzero $\alpha$, regardless of its sign. In contrast, with $f_{b}$ the $\Delta \phi=\pi$ states remain stable when $\alpha>0$ but lose stability when $\alpha<0$. In SH35 the odd solutions play a similar role. These can be stable under homogeneous forcing but become unstable in the presence of the forcing $f_{p}$ with nonzero $\alpha$ and $f_{b}$ with $\alpha<0$. This is also the case for some of the even states in SH35 with $f_{p}$, for example, those between the saddle nodes at $r_{2}$ and $r_{3}$ which become unstable as soon as $\alpha \neq 0$.

In this section we study the dynamics resulting from these instabilities using direct numerical integration. We use the time-stepping scheme ETD4RK [34] with Fourier basis functions in space. The solutions are dealiased according to the degree of the nonlinearity: half the spectrum is removed for $\mathrm{SH} 23$ while two thirds are removed for SH35. The simulations below use 1024 modes.

When $\alpha \neq 0$ time-independent periodic solutions of SH23 have a specific phase relative to the forcing $f_{p}, \Delta \phi=0$ (stable) and $\Delta \phi=\pi$ (unstable). Initial value simulations starting from a periodic initial condition with $\Delta \phi \neq 0$ show that the solution becomes asymmetric as soon as $\alpha \neq 0$ and starts to drift towards the stable, energetically preferred (with respect to $\mathcal{F}$ ) state $\Delta \phi=0$. This is the case with the forcing $f_{b}$ as well: here the preferred state resembles a periodic structure with a defect in the vicinity of the bump. Localized states also drift as soon as translation invariance is broken. Figure 26(a) shows the evolution of an unstable $\Delta \phi=\pi$ state with the forcing $f_{b}$ and $\alpha<0$ : the solution drifts towards a $\Delta \phi=0$ state. This state is not stable, however, and the systems finds a stationary but asymmetric state instead [Figs. 18(b) and 27]. States of this type are generated from the asymmetric rung states as soon as translation invariance is broken $(\alpha \neq 0)$; Fig. 18(b) shows that the dominant peak in the solution is in phase with the imposed bump, but the overall solution is highly asymmetric with respect to this point. We think of solutions of this type as states that would drift in the absence of the bump, but that are trapped or pinned by the bump.

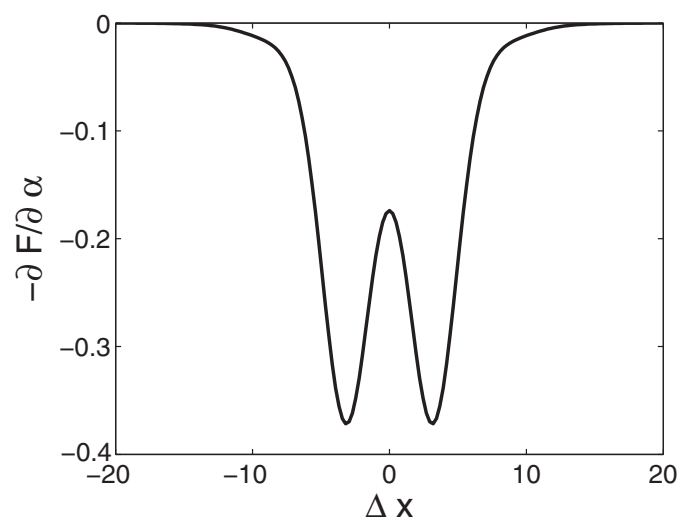

FIG. 27. The quantity $-F_{\alpha}\left(=\frac{1}{2} \int_{-\Gamma / 2}^{\Gamma / 2} r f_{b}(x+\Delta x) u_{0}(x)^{2}\right)$ in $\mathrm{SH} 23$ as a function of the displacement $\Delta x$ of the forcing $f_{b}$ relative to the symmetry point of an $\alpha=0, \Delta \phi=\pi$ localized state with $r=-0.306$ and $N=0.217$, showing the presence of nearby stable asymmetric states corresponding to local minima with $\Delta x \neq 0$. 
(a)

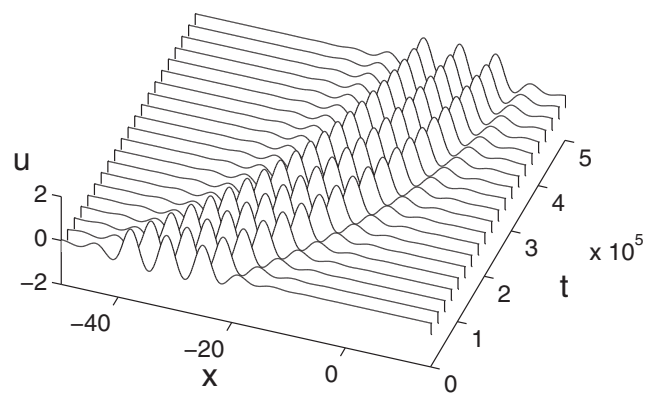

(b)

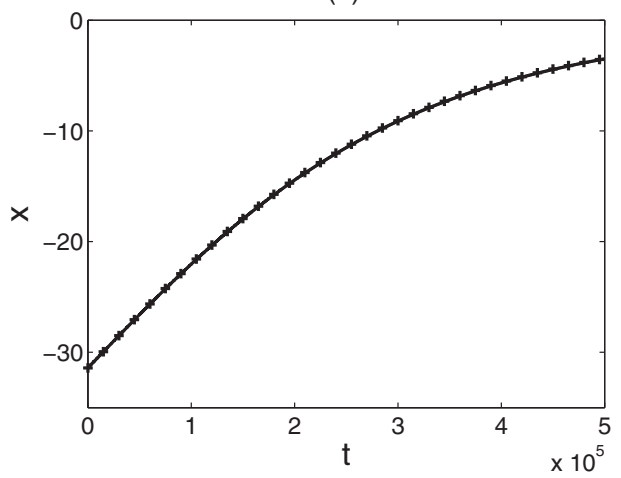

FIG. 28. (a) Time evolution of an unstable $\phi=0$ localized solution in $\mathrm{SH} 23$ with $f_{p}$ forcing and $\alpha=-0.01, \delta=0.05$. (b) Location of the maximum of the solution as a function of $t$. The solid line represents the results of direct numerical simulation while the + signs are computed from the asymptotic result in Eq. (12).

For comparison we show in Fig. 26(c) the corresponding evolution of an unstable odd state in SH35 with the forcing $f_{b}$ and $\alpha<0$. The figure shows that the state evolves into a stable stationary but asymmetric state. Figure 19(b) shows the resulting final state.

We can use these simulations to compare the observed front speed with the prediction Eq. (12). For this purpose we consider the case of SH23 with $f_{p}$ forcing and $\alpha=-0.01$ in the same setting as in Sec. V. We pick a stable in-phase localized solution obtained from numerical continuation and shift it horizontally by $-10 \pi$ to generate an initial condition. Figure 28 shows the time evolution and the location of the maximum of the solution as a function of time. The solid line in (b) is obtained by solving the Swift-Hohenberg equation numerically while the data obtained from Eq. (12) is represented using the + symbol. The result shows excellent agreement between the asymptotic calculation and the full numerical result.

\section{SUMMARY}

In this paper we have described the effects of spatial heterogeneities on the properties of spatially localized structures (LS) in one spatial dimension. For this purpose we have used a modification of the well-studied Swift-Hohenberg equation, focusing on two cases, the quadratic-cubic equation ( $\mathrm{SH} 23$ ) and the cubic-quintic equation (SH35). In each case we applied multiplicative spatial forcing by allowing the parameter $a$ to depend on space. Forcing of this type preserves the homogeneous state $u=0$ while selecting preferred locations for the spatial structures, somewhat in the manner of finite domain boundary conditions [25,26]. We first examined the effects of periodic forcing with wavelength equal to the natural wavelength generated by the Swift-Hohenberg equation. This case corresponds therefore to 1:1 spatial resonance, and the behavior observed is characteristic of strong resonance problems. Specifically, we found that LS in phase with the forcing could be stable, while out-of-phase LS were necessarily unstable. In SH23 the former snake in the normal fashion, while the snaking in the latter is incomplete and results in the formation of two-pulse LS, much as in $\mathrm{SH} 23$ with mixed boundary conditions [25]. In SH35 even parity states also snake but for small forcing do so between four limiting values; with increased forcing the inner limit points merge via a process resembling that identified in Ref. [28] in connection with the breaking of the $u \rightarrow-u$ symmetry of SH35. We have identified an explanation for this behavior and confirmed the predictions by examining the splitting of the saddle nodes as the forcing amplitude $\alpha$ becomes nonzero. In contrast, the odd parity states execute incomplete snaking.

We have also considered the case of an isolated forcing bump on the scale of the natural wavelength of the system and found solutions that are localized at the location of the bump, while others are repelled. We refer to the former as trapped; such states also snake but the snaking branch is now a primary solution branch that evolves, once the domain is full, into an extended defect state, much as occurs in systems with non-Neumann boundary conditions [25]. Finally, we employed periodic forcing with only one period in the (large) domain. Such forcing is locally homogeneous but the effective bifurcation parameter depends on the location. This effect was found to incline the snaking branches present in strictly homogeneous systems, thereby providing an alternative explanation of slanted snaking observed in experiments [12,33,35]. Evidently slanted snaking need not arise only as the result of a conserved quantity as in Refs. [6,7,33] but can also be the result of large scale parameter variation across the experimental system.

Throughout we examined the stability of the localized states we have found, identifying both stable and unstable structures as a function of the model parameters. Numerical simulations were performed to identify the longtime fate of unstable solutions. Structures destabilized via an unstable phase mode become asymmetric and undergo drift, a result of the tilting of the effective potential by the heterogeneous forcing, until such time as they become trapped or reach a region characterized by spatially homogeneous parameters.

Spatial forcing of the Swift-Hohenberg equation has been considered before [36,37] although not in the subcritical regime where trapping of localized states becomes possible. We expect that the results reported here will be of interest to experimentalists working with localized structures, as well as to theorists interested in identifying generic processes resulting from forced symmetry breaking. Problems of this type have numerous applications, from fluid mechanics [38] 
and optics $[39,40]$, to reaction-diffusion models [41,42] and recent work on models of desertification $[43,44]$. In addition, localized bump forcing may serve as a model of a persistent perturbation, such as may be applied by a focused optical probe in an optics experiment, in contrast to instantaneous perturbations applied by turning the probe rapidly on and off. The latter example leads to initial value evolution with a different initial condition; the former can lead to persistent structures trapped by the inhomogeneity whose properties may be tailored by appropriate shaping of the bump profile [45].

\section{ACKNOWLEDGMENTS}

We are grateful to D. Avitabile and U. Thiele for helpful discussions. This work was supported by National Science Foundation under the grant DMS-1211953.
[1] K. Ghorayeb and A. Mojtabi, Phys. Fluids 9, 2339 (1997).

[2] S. Blanchflower, Phys. Lett. A 261, 74 (1999).

[3] O. Batiste, E. Knobloch, A. Alonso, and I. Mercader, J. Fluid Mech. 560, 149 (2006).

[4] A. Bergeon and E. Knobloch, Phys. Fluids 20, 034102 (2008).

[5] I. Mercader, O. Batiste, A. Alonso, and E. Knobloch, J. Fluid Mech. 667, 586 (2011).

[6] D. Lo Jacono, A. Bergeon, and E. Knobloch, J. Fluid Mech. 687, 595 (2011).

[7] C. Beaume, A. Bergeon, H.-C. Kao, and E. Knobloch, J. Fluid Mech. 717, 417 (2013).

[8] R. Richter and I. V. Barashenkov, Phys. Rev. Lett. 94, 184503 (2005).

[9] U. Bortolozzo, M. G. Clerc, and S. Residori, New J. Phys. 11, 093037 (2009).

[10] T. M. Schneider, J. F. Gibson, and J. Burke, Phys. Rev. Lett. 104, 104501 (2010).

[11] M. Avila, F. Mellibovsky, N. Roland, and B. Hof, Phys. Rev. Lett. 110, 224502 (2013).

[12] H.-G. Purwins, H. U. Bödeker, and S. Amiranashvili, Adv. Phys. 59, 485 (2010).

[13] C. Etrich, U. Peschel, and F. Lederer, Phys. Rev. Lett. 79, 2454 (1997).

[14] D. V. Skryabin, Phys. Rev. E 60, 3508 (1999).

[15] J. Burke, S. M. Houghton, and E. Knobloch, Phys. Rev. E 80, 036202 (2009).

[16] J. Burke and E. Knobloch, Chaos 17, 037102 (2007).

[17] A. Alonso, O. Batiste, E. Knobloch, and I. Mercader, in Localized States in Physics: Solitons and Patterns, edited by O. Descalzi, M. G. Clerc, S. Residori, and G. Assanto (Springer, Berlin, 2010), pp. 109-125.

[18] J. Burke and E. Knobloch, Phys. Lett. A 360, 681 (2007).

[19] P. D. Woods and A. R. Champneys, Physica D 129, 147 (1999).

[20] M. Beck, J. Knobloch, D. J. B. Lloyd, B. Sandstede, and T. Wagenknecht, SIAM J. Math. Anal. 41, 936 (2009).

[21] E. J. Doedel, A. R. Champneys, F. Dercole, T. Fairgrieve, Y. Kuznetsov, B. Oldeman, R. Paffenroth, B. Sandstede, X. Wang, and C. Zhang, AUTO-07P: Continuation and Bifurcation Software for Ordinary Differential Equations (Concordia University, Montreal, 2008).

[22] J. Burke and E. Knobloch, Phys. Rev. E 73, 056211 (2006).
[23] A. Bergeon, J. Burke, E. Knobloch, and I. Mercader, Phys. Rev. E 78, 046201 (2008).

[24] D. Lo Jacono, A. Bergeon, and E. Knobloch, Fluid Dyn. Res. 44, 031411 (2012).

[25] S. M. Houghton and E. Knobloch, Phys. Rev. E 80, 026210 (2009).

[26] G. Kozyreff, P. Assemat, and S. J. Chapman, Phys. Rev. Lett. 103, 164501 (2009).

[27] D. Avitabile and H. Schmidt (unpublished).

[28] S. M. Houghton and E. Knobloch, Phys. Rev. E 84, 016204 (2011).

[29] E. Makrides and B. Sandstede, Physica D 268, 59 (2014).

[30] I. Mercader, O. Batiste, A. Alonso, and E. Knobloch, Phys. Rev. E 80, 025201(R) (2009).

[31] G. Dangelmayr, J. Hettel, and E. Knobloch, Nonlinearity 10, 1093 (1997).

[32] P. Hirschberg and E. Knobloch, J. Nonlinear Sci. 7, 537 (1997).

[33] J. H. P. Dawes, SIAM J. Appl. Dyn. Syst. 7, 186 (2008).

[34] S. M. Cox and P. C. Matthews, J. Comp. Phys. 176, 430 (2002).

[35] W. J. Firth, L. Columbo, and A. J. Scroggie, Phys. Rev. Lett. 99, 104503 (2007).

[36] R. Manor, A. Hagberg, and E. Meron, Europhys. Lett. 83, 10005 (2008).

[37] R. Manor, A. Hagberg, and E. Meron, New J. Phys. 11, 063016 (2009).

[38] E. L. Koschmieder, Adv. Chem. Phys. 26, 177 (1974).

[39] S. Barbay, Y. Ménesguen, X. Hachair, L. Leroy, I. Sagnes, and R. Kuszelewicz, Opt. Lett. 31, 1504 (2006).

[40] P. Parra-Rivas, D. Gomila, M. A. Matías, and P. Colet, Phys. Rev. Lett. 110, 064103 (2013).

[41] Y. Nishiura, T. Teramoto, X. Yuan, and K.-I. Ueda, Chaos 17, 037104 (2007).

[42] Y. Nishiura, T. Teramoto, and X. Yuan, Comm. Pure Appl. Anal. 11, 307 (2012).

[43] M. Tlidi, R. Lefever, and A. Vladimirov, in Dissipative Solitons: From Optics to Biology and Medicine, edited by N. Akhmediev and A. Ankiewicz, Lecture Notes in Physics 751 (Springer, New York, 2008), pp. 381-402.

[44] E. Meron, Ecol. Model. 234, 70 (2012).

[45] A. Yochelis, E. Knobloch, Y. Xie, Z. Qu, and A. Garfinkel, Europhys. Lett. 83, 64005 (2008). 